\title{
La agricultura social en Cataluña: diagnosis de un fenómeno emergente
}

Social Farming in Catalonia: diagnosis of an emerging phenomenon

\section{Carles Guirado González}

carlesguirado@gmail.com

\section{Natàlia Valldeperas Belmonte}

nataliavalldeperas@gmail.com

\author{
Ana Vera Martín \\ ana.vera@uab.cat \\ Antoni F. Tulla Pujol \\ antoni.tulla@uab.cat \\ Departament de Geografia \\ Universitat Autònoma de Barcelona (España)
}

\section{Resumen}

La Agricultura Social (AS) utiliza la participación de colectivos en riesgo de exclusión social en actividades agrarias con el objetivo de mejorar sus condiciones de vida, incluirlos en la sociedad, darles una oportunidad laboral y empoderarlos. Este ámbito de la economía social, según los agentes clave del sector, ayuda al mantenimiento de las pequeñas explotaciones agrarias, contribuyendo al desarrollo local y a la cohesión territorial desde la óptica de la multifuncionalidad agraria. La AS, en sus diversas tipologías y modelos, es un sector presente en diferentes países de Europa desde hace más de 30 años. En Cataluña, ámbito de nuestro estudio, ha iniciado su andadura desde finales del siglo XX. Este artículo plantea algunos de los elementos teóricos y conceptuales que giran en torno a la AS, presenta las principales características del sector en Cataluña, analizando la tipología de las entidades existentes, las actividades y los principales 
grupos de interés (stakeholders) que configuran el sector desde una perspectiva económica, social y ambiental, para, por último, realizar una diagnosis del sector y esbozar algunos de los retos a los que se enfrenta. Para ello se han utilizado diferentes métodos cuantitativos y cualitativos, combinados con el fin de ofrecer una radiografía lo más exhaustiva posible de un fenómeno.

Palabras clave: agricultura social; desarrollo local; multifuncionalidad; exclusión social; Cataluña.

\begin{abstract}
Social Farming (SF) engages groups at risk of social exclusion in agricultural activities with the aim of including them in society, providing them with job opportunities, and empowering them. This social economic field, according to key agents, helps to sustain small farms, which contributes to local development and territorial cohesion from the viewpoint of multifunctional agriculture. SF, in its various types and models, has already existed in different countries of Europe since more than 30 years. In Catalonia, scope of our study, it is a phenomenon that has begun its development in late twentieth century. In this paper, we show some theoretical and conceptual elements about SF, describing the types of entities that exist in Catalonia, their activities, and the main stakeholders that shape the SF sector from the economic, social and environmental perspectives. Finally, we make a diagnosis about this social and economic sector and sketch out some future challenges. In order to conduct a thorough diagnosis of the phenomenon, we combined quantitative and qualitative methodology.
\end{abstract}

Key words: social farming; local development; multifuncionality; social exclusion; Catalonia.

\title{
1 Introducción
}

La AS podría definirse a grandes rasgos como un proceso de inclusión social y empoderamiento de colectivos en riesgo de exclusión social a través de la ocupación en actividades agrarias (o derivadas) y/o en la transformación de sus productos mediante procesos artesanales, que permitan conectar al individuo con la naturaleza, el origen del producto, el ciclo de vida de los organismos vivos y con los beneficios de los procesos de manipulación manual. Así pues, la AS es una actividad que añade nuevos significados a la práctica agrícola más allá de las cuestiones productivas, proporcionándole una función social concreta con el objetivo de aumentar la calidad de vida de las personas en riesgo de exclusión social. Esta práctica resulta una oportunidad de ocupación para los grupos de mayor fragilidad social, los cuales pueden incluirse en la sociedad mediante el trabajo agrícola y al mismo tiempo mejorar otros aspectos importantes como su salud física y mental y recuperar su dignidad personal. Los procesos de inclusión de estos colectivos a través de la AS se basan en la creación de lugares de trabajo o de formación, que a menudo van 
acompañados de un conjunto de medidas terapéuticas, de inserción laboral y/o de apoyo psicológico o sanitario para garantizar los beneficios del proyecto sobre las personas usuarias.

Generalmente, la AS es un fenómeno presente en espacios agrícolas de las áreas rurales y periurbanas de los países europeos y norteamericanos, aunque se está empezando a expandir significativamente en las áreas urbanas (Lohrberg, 2016). Sin embargo, es en los territorios más o menos remotos donde la implantación de proyectos de AS tienen mayores beneficios, facilitando la creación de servicios sociales y de salud, al mismo tiempo que aportan beneficios a la población local, favoreciendo la creación de valor añadido mediante actividades de transformación y comercialización de productos agroalimentarios, aumentando la cohesión social y promoviendo una economía en pro del bien común (Guirado, Badia, Tulla, Vera \& Valldeperas, 2014).

A grandes rasgos, podemos afirmar que el fenómeno de la AS es bastante heterogéneo, ya que las entidades que desarrollan estas prácticas, su forma jurídica, el financiamiento y la colaboración entre las instituciones públicas y privadas dependen de cada territorio y están sujetas a las características del sistema de bienestar, el sistema de salud y las políticas sociales de cada país (Tulla, Vera, Badia, Guirado \& Valldeperas, 2014). La AS es un fenómeno presente en todo el continente europeo, donde los diferentes modelos de implementación varían substancialmente en función de la organización del estado y se fundamentan en los sistemas de regulación de la atención socio-sanitaria, la tipología de los actores involucrados, las condiciones de bienestar de las sociedades, la financiación de los proyectos y las diferentes formas de su gestión (Esping-Anersen, 1996; Di lacovo \& O'Connor, 2009; O’Connor, Lai \& Watson, 2010; Guirado et al., 2013).

El objetivo principal de este artículo es mostrar cómo la AS resulta ser un actividad estratégica para el desarrollo local sostenible, tanto social, económica como ambientalmente, y como esta se implanta en un territorio concreto. En primer lugar, realizaremos una aproximación teórica y conceptual del fenómeno y presentaremos las diversas variantes de la AS en Europa, mediante la revisión de la literatura científica. Posteriormente, analizaremos la evolución y dinámica del fenómeno en Cataluña mediante la recogida de datos estadísticos, las entrevistas a agentes clave del sector y la diagnosis realizada a partir de toda la información recogida. Finalmente, el análisis DAFO y el flujograma, explicados más adelante, nos permitirán apuntar algunos de los retos estratégicos a los que debe hacer frente el sector.

\section{Marco teórico y conceptual}

\section{1 "Nuevas" prácticas locales para "nuevos" escenarios globales: contexto socioeconómico, dinámicas territoriales y pautas socioculturales}

Desde hace siglos, la mutualidad y las actividades en beneficio de lo colectivo han aportado una función eminentemente social al trabajo agrícola que ha favorecido la cohesión social de la 
población rural y la atención de los grupos más vulnerables en estos territorios. En la época medieval ya se conocía la importancia del contacto con los espacios naturales como herramienta terapéutica, que añadía un valor rehabilitador y una clara función integradora a la actividad agraria. Esta práctica se daba sobre todo en espacios de índole religiosa, como son los monasterios u hospitales, donde era habitual la existencia de jardines y huertos de carácter terapéutico u ocupacional (Gerlach-Spriggs et al., 2004; Stigsdotter et al., 2011), en las que coexistían la función social junto con la función productiva. Así pues la integración de estos colectivos mediante la agricultura es una práctica utilizada de antiguo (Di lacovo \& O’Connor, 2009), lo cual convierte la AS en una actividad de largo recorrido.

Los beneficios observados mediante la interacción entre las personas con trastorno mental y los elementos naturales fueron destacados a inicios del siglo XIX por algunos profesionales del sector de la salud, entre ellos el psiquiatra americano Benjamin Rush. Estas apreciaciones impulsan la aparición de la horticultura terapéutica, una variante del concepto actual de AS, aplicada por Rush en distintos centros de salud mental (Relf, 2006). A mediados del siglo XX el trabajo agrario se difunde hacia ámbitos como el de la inserción sociolaboral y la terapia ocupacional (Di lacovo \& O'Connor, 2009), siendo el Reino Unido donde aparecen los primeros centros de trabajo protegido en los que la actividad agrícola se emplea para empoderar a los colectivos más vulnerables. Así pues, estos centros de carácter religioso promovidos mayoritariamente por obras de beneficencia o charities, son un factor clave en los orígenes del fenómeno actual de la AS en Europa.

El mayor impulso de las prácticas de AS se produce en las últimas décadas del siglo XX pudiéndose comprobar un crecimiento considerable de nuevas iniciativas así como la creación de nuevas organizaciones territoriales que la estructuran (Di lacovo \& O’Connor, 2009). En los países del sur de Europa gran parte de las iniciativas surgen desde la sociedad civil, con el doble objetivo de solucionar las necesidades específicas de determinados colectivos, y como respuesta a las carencias del sistema de atención sociosanitaria y de bienestar. De este modo el Tercer Sector Social (TSS) genera alternativas a estas deficiencias en una coyuntura de crisis socioeconómica en la que la exclusión afecta cada vez más a un mayor número de personas (Di lacovo et al., 2014). Además, los cambios surgidos en esta coyuntura socioeconómica se traducen en la aparición de nuevas pautas socioculturales como la salud, los sistemas de bienestar, la producción y consumo agroalimentario, y el desarrollo rural sostenible dando lugar a la manifestación de actividades innovadoras, como es la AS.

La conceptualización de las nuevas políticas y prácticas agroalimentarias emergentes en el ámbito internacional, que aparecen en el marco de la producción y el consumo de productos agroalimentarios, toma un nuevo impulso a finales del siglo XX gracias al trabajo de algunos académicos (Murdoch \& Miele, 1999; Renting et al., 2003; Ploeg, 2008). En estas aportaciones 
se define un nuevo paradigma en la producción de los alimentos y de desarrollo rural, en el que se revaloriza la producción agroalimentaria de proximidad. El giro hacia la calidad o quality turn (Goodman, 2004), se aleja de los valores relacionados con la etapa productivista (Bowler, 1996; Lockwood, 1999), dando más importancia a la calidad del producto que a la cantidad con que se produce. La redefinición de los usos y funciones del territorio rural, de los sistemas agroalimentarios, de la producción del espacio y del papel de la agricultura en la sociedad actual se explican, en parte, por este cambio de paradigma, el cual se encuadra en la etapa posproductivista (Guinjoan et al., 2016). Al mismo tiempo aparece el movimiento agroecológico (López \& Fernández, 2003; Sevilla, 2006; Aurélie, 2007; Ploeg, 2008; Vivas, 2010; Monllor, 2013), un nuevo paradigma en el que surge un campesinado (Ploeg, 2010), con nuevos valores y formas de producir en el que se elige un modelo agroalimentario con más justicia social, sustentado en otras relaciones económicas y con mayor respeto por el medio ambiente (Petrini, 2003; Duch, 2010).

Actualmente, la crisis global en la que vivimos ha acarreado un importante cambio de valores y en las pautas socioculturales de la población en diferentes ámbitos (Bauman, 2013). En el sector agroalimentario, concretamente, es difícil controlar los procesos de producción industrial de los alimentos. La inseguridad alimentaria asociada a ello ha provocado una modificación en las pautas de consumo de ciertos grupos sociales (Guidonet, 2010; Medina, 2010; Levenstein, 2012). Por ello, el consumo de productos ecológicos ha sido una opción escogida, en algunos casos por motivos ideológicos y en otros por salud, por parte de ciertos grupos sociales, con el fin de poder recuperar métodos de producción más artesanales, no tanto en la implementación del conocimiento agroalimentario, sino más en la salud, proximidad y calidad (DuPuis \& Goodman, 2005; Miele, 2006). La producción ecológica permite conocer lo que se está comiendo, teniendo ello efectos positivos directos en la salud de las personas y en una configuración del modelo agroalimentario que favorece a un entorno más justo socialmente, con mayor respeto hacia el medio ambiente y con una mayor calidad final del producto (Carbone et al., 2007; Schneider, 2008; López-Moreno et al., 2016).

En este sentido, la AS contribuye al cambio social y económico en el sector agroalimentario, en tanto que los proyectos se configuran como un importante agente de creación y fomento de redes alimentarias alternativas (Sánchez Hernández, 2009). Las pautas de consumo actuales de ciertos segmentos de la sociedad, basadas en la adquisición de alimentos buenos, sanos y justos, junto con las preferencias ideológicas y el descontento con el modelo agroindustrial han llevado a las personas promotoras de las experiencias de AS a desarrollar modelos de producción social, ambiental y económicamente más sostenibles, que apuestan por una agricultura orgánica que cree oportunidades de ocupación, formación, terapia y/o rehabilitación para persones socialmente vulnerables, además de forjar nuevos modelos de relación entre el productor y el consumidor, 
basados en la proximidad, la confianza y la solidaridad. Esta dimensión y función de la AS colabora estrechamente con el desarrollo local, especialmente en los territorios donde se ubican los proyectos, en los que se suma a otras experiencias locales para fortalecer la creación de redes alimentarias alternativas y la transición local agroecológica.

Del mismo modo que en el sector agroalimentario, la supremacía del modelo biomédico y del sistema de atención sociosanitario convencional y hegemónico está siendo cada vez más cuestionado (Astin, 1998; Ballvé, 2003). Se empieza a poder demostrar científicamente la relación entre un empeoramiento en el estado de la salud mental y física de las personas, vinculadas directamente a algunos tratamientos de la medicina convencional, y el abuso de fármacos (Bachrach, 1996; Palinkas et al., 2000; Lamb \& Bacharch, 2001). En este contexto, una parte creciente de la sociedad apuesta por otro tipo de medicación y/o tratamientos, algunos de ellos con especial incidencia en el uso de los elementos naturales para abordar ciertas patologías y estados mentales (Stigsdotter et al., 2011), en los que la prioridad está en el bienestar y la promoción de la salud de las personas pudiendo ofrece al usuario una atención más integral y personalizada (Astin, 1998). Además de esto, podemos añadir que las prácticas que utilizan el elemento natural como herramienta clave para los procesos de rehabilitación promueven la mejora en el estado mental y físico de las personas usuarias, el bienestar emocional, un estilo de vida más saludable y a la vez mejoran la relación con la atención sociosanitaria (Townsend, 2006; Sempik et al., 2010; Bragg, 2014; Kuo, 2015).

Para finalizar, hay que resaltar la crisis que experimentan los sistemas de atención social, de salud y de bienestar de algunos países europeos en el contexto de crisis financiera. Los elevados costes del sistema público de salud aumentan rápidamente debido a factores como el envejecimiento de la población, la creciente presencia de enfermedades degenerativas y crónicas y de hábitos de vida poco saludables. Todo esto, junto con el endeudamiento de los estados, explica el colapso en el que se encuentran muchos de los servicios de atención sociosanitaria actuales. La desinstitucionalización del sector sanitario y de atención, la privatización y la descentralización son algunas de las repercusiones de la situación descrita anteriormente, y que se puede interpretar como una estrategia para reducir los gastos para las arcas públicas que requiere mantener el sistema de salud pública (Maarse, 2006). La descentralización de las competencias en materia de atención de personas con fragilidades, a entidades del TSS o del sector privado, con la reducción de medicamentos subvencionados, la aplicación de sistemas de copago de los tratamientos, y la externalización de servicios sociosanitarios a empresas privadas proveedoras de servicios de salud (Saltman et al., 2006).

En este sentido, la AS se presenta como un elemento estratégico para ofrecer soluciones múltiples a necesidades presentes en la sociedad actual, y con ellas subsanar las carencias de las políticas públicas y la disconformidad con enfoques neoliberales en distintos ámbitos, tanto en la esfera de 
los servicios sociales y la atención sociosanitaria como en la del desarrollo rural y local, de las políticas agrarias y del modelo agroalimentario. La AS aparece también como una oportunidad para llevar a cabo una mayor diversificación de actividades en el ámbito rural y periurbano, donde el valor productivo va junto a la función social, destinada a empoderar a personas en riesgo de exclusión.

\subsection{La Agricultura Social, una aproximación conceptual}

Para elaborar este apartado del artículo se ha realizado la revisión de las principales publicaciones científicas que versaban sobre nuestro objeto de estudio, con el objetivo de configurar un cuerpo teórico y conceptual sobre la AS. Se han consultado revistas académicas especializadas, informes derivados de proyectos europeos -como el proyecto SoFar: Social Services in Multifunctional Farms $^{1}$ o el proyecto Green Care in Agriculture (Cost Action 866) ${ }^{2}$-, artículos y publicaciones de carácter divulgativo sobre la AS en Europa, España y Cataluña. La participación en varios congresos nacionales e internacionales nos ha permitido ampliar el conocimiento sobre esta práctica, enriquecer la investigación y aportar nuevos enfoques a un marco teórico en constante revisión. En este proceso, hemos podido observar una cierta escasez de investigaciones sobre la AS en España y Portugal, lo cual también refuerza el interés de nuestro estudio. Las publicaciones referentes al tema en el ámbito académico ibérico son escasas y hay pocos grupos de investigación que estén desarrollando alguna línea de pesquisa en la órbita de la AS. En España podemos destacar, en primer lugar, los trabajos realizados en el marco del Programa de Soberanía Alimentaria para la Lucha contra el Hambre y el Desarrollo Local impulsado por el Área de Cooperación y Solidaridad de la Universidad de Córdoba, que ha llevado a cabo un diagnóstico comarcal de las experiencias de AS en la campiña sur de esta ciudad (Lamarca, 2015; Lamarca ef al., 2016), y, en segundo lugar, las investigaciones del equipo de la Aliança per la Sobirania Alimentària de Cataluña, que analizan la práctica de la AS como un mecanismo para contribuir a la soberanía alimentaria y reducir la pobreza alimentaria de ciertos colectivos en Cataluña (Pomar, Tendero, 2015). También aparecen otras investigaciones y artículos de divulgación que han ido abordando el tema desde múltiples puntos de vista, como la sostenibilidad ecológica (GarcíaLlorente et al., 2015), el Green Care (Iranzo-Garcia et al, 2015) o los huertos comunitarios (Fernández \& Morán, 2011). En el caso de Portugal, son conocidos los trabajos de Mourão y Brito (2013), sobre las características y los beneficios de la horticultura social y terapéutica; de Firmino (2011), sobre la situación de la AS en Portugal; y de Da Silva (2015), que realiza un estudio aplicado de los beneficios económicos, sociales y ambientales de los huertos sociales ecológicos de un municipio del norte de Portugal, cercano a Porto. Pese a que cada vez son más los

1 Más información en httpp://sofar.unipi.it/

2 Más información en http://www.umb.no/greencare/ 
investigadores y las instituciones que muestran interés por analizar este fenómeno social en auge, aún queda mucho por hacer en este campo. Algunos enfoques se aproximan de forma indirecta al tema, a través del estudio de los espacios agrarios periurbanos (Matarán, 2013), la producción ecológica y la soberanía alimentaria (Cuéllar et al., 2013).

La revisión de los principales trabajos realizados en Europa nos ha permitido observar que la AS es un fenómeno extendido y consolidado en muchos países del continente, en la que la actividad agraria, la atención sociosanitaria y las políticas sociales se unen para dar respuestas innovadoras a los colectivos en riesgo de exclusión social. Los agentes que desarrollan estos proyectos son mayoritariamente cooperativas, asociaciones, fundaciones privadas, centros de salud y obras de beneficencia relacionadas a movimientos religiosos, entre otras organizaciones $\left(\mathrm{O}^{\prime} \mathrm{Connor}\right.$ et al., 2010). A partir del análisis bibliográfico realizado hemos encontrado un importante número de definiciones y distintos términos que tienen como objetivo tratar las diversas características de la AS.

Actualmente hay importantes diferencias en el desarrollo de la AS en Europa, ya que cada país tiene sus mecanismos de regulación, sus organizaciones de apoyo y ha implementado sus propias leyes. Cada estado ha adaptado este fenómeno a los sistemas de bienestar, a los modelos de atención sociosanitario, a los programas de desarrollo rural y a las políticas sociales. No obstante, y como ya apunta Di lacovo (2010), hemos detectado que existen grandes modelos de implementación, y que marcan una diferenciación clara entre los países del centro y el norte y los del sud de Europa, por lo que hace referencia tanto a los estadios de desarrollo de la AS como a la tipología de las experiencias. En los países del norte y centro de Europa, los gobiernos tiene un papel predominante en la regulación y el desarrollo de la AS, según la esencia del fenómeno y el estado de madurez del mismo (Di lacovo, 2010; Guirado et al., 2013; Tulla et al., 2015). El Estado fija directrices claras para el desarrollo de dicha actividad, fomenta así la creación de instituciones para apoyar al desarrollo de la AS, se establecen líneas de financiación concretas para las personas promotoras de experiencias de AS y para las entidades proveedoras de la atención sociosanitaria y se definen programas de subvenciones públicas para los usuarios. En cambio, en los países del sud de Europa - menos en el caso de Italia, donde el Parlamento aprobó una ley específica en agosto de $2015^{3}$ - el diferente grado de desarrollo emergente del fenómeno hace que el estado ocupe un lugar subsidiario en el desarrollo de la AS, un hecho que repercute en la creación de nuevas iniciativas en el TSS y otras entidades de la sociedad que impulsan el sector y el establecimiento de redes de ayuda, como es el caso de España (Guirado et al., 2014), Portugal (Firmino, 2011; Da Silva, 2015) y Grecia (Galiakis, 2014). Así pues, una misma actividad puede adoptar diversas formas en contextos territoriales distintos, creando al mismo tiempo múltiples

3 Disposizioni in materia di agricoltura sociale. Gazzetta Ufficiale de la Repubblica Italina, Legge 18 agosto 2015 , n. 141. Retrieved from http://www.gazzettaufficiale.it/eli/id/2015/09/8/15G00155/sg 
conceptos para configurar la realidad de un fenómeno complejo y heterogéneo (Hassink, 2003; Hassink \& Van Dijk, 2006; Hine et al., 2008; Di lacovo \& O'Connor, 2009; Sempik, Hine \& Wilcox, 2010).

Green care (GC) es uno de los conceptos que generalmente se relaciona con la AS. Este recoge un amplio grupo de actividades que tienen en común el uso de elementos naturales para la promoción y el mantenimiento del bienestar físico, mental, social y educacional de las personas (Haubenhofer et al., 2010; Sempik, Hine \& Wilcox, 2010). Green care in agriculture (GCA) se utiliza para denominar aquel tipo de prácticas que aparecen ubicadas en el entorno natural y relacionadas con el trabajo agrario, eso significa el uso de fincas agrarias como elemento para el impulso de la salud física y mental de las personas beneficiarias. Esta práctica incluye un amplio abanico de experiencias, de tipologías muy variadas, que desarrollan su actividad principalmente en el ámbito terapéutico, de la rehabilitación, la promoción de la salud y la atención sociosanitaria, atendiendo a un amplio espectro de grupos sociales, centrándose en aquellos sectores con más fragilidades (Dessein et al., 2013).

Care farming (CF) es otro de los conceptos asociados a la AS que empezó a ser utilizado en el Reino Unido a inicios del siglo XXI, adquiriendo el apelativo que ya se utilizaba en Holanda para referirse a actividades similares (Leck et al., 2014). El CF se define como el uso de explotaciones agrarias convencionales y de los paisajes agrícolas como elementos clave para promover la salud física y mental de las personas (Hine et al., 2008). El ámbito donde se desarrolla el CF es principalmente el terapéutico, aunque también existen algunas experiencias en el ámbito de la educación, la terapia ocupacional o los programas de integración social (Hasssink \& Van Dijk, 2006). El objetivo se basa en la creación de oportunidades de desarrollo personal y de rehabilitación con personas vulnerables (Sempik et al., 2010), entre ellas personas con discapacidad y/o trastorno mental, con algún tipo de adicción, con dificultades de aprendizaje o abandono prematuro de los estudios, personas sin hogar o personas del ámbito penitenciario Un elemento diferenciador de otros conceptos es que la explotación agraria tradicional (farm) actúa como centro proveedor de estos servicios de atención sociosanitaria, donde las personas usuarias reciben un cuidado integral y realizan actividades propias del trabajo agrario, la gestión del territorio y el mantenimiento de la finca (Leck et al., 2014). Las personas usuarias de las care farms pagan una cantidad variable para acceder a los servicios que se ofrecen en estos centros. En determinadas ocasiones, según apuntan Dessin et al. (2013), es el sistema público de salud el organismo encargado de financiar, ya sea parcialmente, estas actividades o bien se paga mediante la afiliación a mutuas privadas de salud, siendo una configuración que puede variar en función de la organización de la actividad de cada territorio. Di lacovo y O’Connor (2009) apuntan que la provisión del cuidado de los usuarios en las care farms se lleva a cabo en presencia de personal cualificado que vela por una evolución del proceso terapéutico satisfactoria. En algunos casos, 
como destaca Leck et al. (2014) en su explicación sobre UK, los propios care farmers son los profesionales que poseen una experiencia y formación especializada en el campo de la atención sociosanitaria que deciden combinarla con el trabajo agrario por motivación personal y/o profesional.

Por último, presentaremos el concepto de AS, derivado del término inglés Social Farming, aproximándonos a las definiciones obtenidas según los diferentes autores que han abordado este término. De manera genérica, la AS considera aquellos elementos que incluyen actividades y servicios terapéuticos, de inclusión, de rehabilitación, educativos y de formación y de inserción laboral mediante los recursos agrarios con la finalidad de promover la salud, principalmente en áreas rurales y periurbanas (Di lacovo \& O’Connor, 2009). Según otras definiciones del término podemos definir la AS como el uso de explotaciones agrarias convencionales y paisajes agroforestales para la promoción del bienestar mental y físico de las personas (Hassink, 2003; Hassink \& Van Dijk, 2006; Hine et al., 2008), ofreciendo importantes beneficios en el ámbito de la salud, social, educativo y laboral a través de la agricultura, destinada a personas en riesgo de exclusión (Sempik, Hine \& Wilcox, 2010). Otros autores como Finoula y Pascale (2008) aportan nuevos elementos al concepto, y consideran que la AS abarca un conjunto de prácticas que compaginan la atención directa a colectivos socialmente vulnerables y el trabajo en fincas agrícolas. Estos colectivos son principalmente personas con discapacidad, personas internas o procedentes de centros penitenciarios, jóvenes, personas inmigradas, personas con problemas de adicción, niños y niñas, gente mayor, mujeres, entre otros, y todos ellos se caracterizan por una ausencia de empoderamiento o emancipación. Este elemento es clave en la conceptualización de la AS, acotando el ámbito de actuación al trabajo agrario, y poniendo el acento en el empoderamiento de aquellas personas que se encuentran en una situación de marginalidad.

Al analizar los diferentes términos se observan algunas diferencias que permiten establecer ciertos límites entre ellos, aun con la dificultad evidente que conlleva delimitar conceptos tan cercanos. Para empezar, podemos mostrar que en las prácticas del GCA y el CF la promoción de la salud aparece como un elemento clave. Así pues, alguno de los elementos que determinan la naturaleza de estas prácticas es el hecho que la actividad ocurra en el contexto de una finca agraria y en provisión con la atención sociosanitaria (care). En cambio, las experiencias de AS se centran más en la producción agroalimentaria de calidad, con el fin de crear proyectos empresariales económicamente viables que tienen como objetivo la inserción sociolaboral, la terapia o la educación de colectivos en riesgo de exclusión social.

Un elemento clave en las experiencias de AS, como ya hemos comentado anteriormente, es el empoderamiento de los colectivos en riesgo de exclusión, mejorando su calidad de vida y promoviendo el desarrollo de su confianza en sus propias habilidades y favoreciendo su participación social, económica y política. En la mayoría de casos el empoderamiento se consigue 
a partir de la inserción sociolaboral, utilizando el trabajo en la agricultura o sectores derivados como vehículo para conseguirlo. En determinadas ocasiones se les ofrece no solamente la ocupación de un lugar de trabajo sino la oportunidad que formen parte de un proyecto colectivo y que participen en la toma de decisiones de la entidad, una contribución importante para su emancipación. Otras diferencias subyacen en los mecanismos y características de acceso al servicio, en el CF o el GCA las personas beneficiarias pagan por los servicios asistenciales, ya sea de manera total o parcial en función de la regulación de cada país y de la situación individual de la persona usuaria, considerando esta actividad como un servicio de salud. En la mayoría de los casos, las personas beneficiarias no forman parte de la organización, y únicamente reciben sus servicios durante un tiempo concreto, abandonado dicha actividad una vez esta se termina. En cambio, se observa que en las experiencias de AS el trabajo con los colectivos es más continuado en el tiempo, con mayor recorrido, y con una atención más integral, abastando diferentes ámbitos de su vida cotidiana, y no únicamente como un servicio de atención sanitaria. Además, en la mayoría de los casos, perciben un salario por su actividad.

Pese a todo, son evidentes las similitudes que existen entre los conceptos presentados, aunque las personas expertas en el tema no han llegado a un consenso claro sobre las diferencias entre estos términos ni cuáles son sus límites. Algunos autores observan que todos estos conceptos se refieren a un fenómeno único y responden a diferentes adaptaciones terminológicas según cada contexto territorial (Dessein et al., 2013; Leck et al., 2014).

Las aportaciones realizadas por los distintos autores consultados han sido el punto de partida para elaborar una definición propia de AS. Esto nos ha servido como marco teórico y al mismo tiempo ha sido útil para aportar más claridad sobre la condición de estas prácticas, concretamente en relación al término de AS. Así pues, definimos que la AS es una serie de experiencias que utilizan los recursos locales, agrarios y/o naturales, para mejorar la salud, la educación, la inserción sociolaboral y el empoderamiento de colectivos en riesgo de exclusión social, en que los beneficiarios son las personas con necesidades laborales específicas (personas con discapacidad, en situación de desempleo de larga duración, en situación de pobreza o procedentes o internas en centros penitenciarios), con necesidad de procesos terapéuticos o de rehabilitación (personas con algún tipo de adicción, mujeres víctimas de violencia de género, personas con trastorno mental, personas con estrés postraumático, entre otros), con necesidades específicas de aprendizaje (niños/as, jóvenes, gente mayor) o bien otros colectivos con fragilidades sociales (personas inmigradas, personas sin hogar, etc.) (Guirado et al., 2014). Hay otro factor importante a resaltar como es la dimensión territorial de la AS, ya que las prácticas que se inscriben en ella contribuyen, a parte de la mejora de la calidad de vida de la personas, a la creación de nuevas estrategias de desarrollo local, promueven la creación de alianzas entre agricultura y atención sociosanitaria y generan nuevos modelos de asistencia y de bienestar. Además, impulsan la creación de nuevos 
servicios para las personas en áreas rurales -territorios donde la dotación de los mismos es bastante inferior a las áreas urbanas- y fomenta una agricultura de proximidad, ecológica y de calidad, con un valor añadido de justicia social (Di lacovo et al., 2013).

\section{Metodología}

La investigación que se presenta en este artículo es parte de los resultados del Equipo de Investigación en Agricultura Social (ERAS) del Departament de Geografia de la Universitat Autònoma de Barcelona obtenidos en los últimos cinco años. ${ }^{4}$ Este trabajo se estructura en tres grandes etapas metodológicas que explicaremos a continuación.

\subsection{Análisis cuantitativo: creación de una base de datos}

En primer lugar, se llevó a cabo la elaboración de una base de datos con el fin de poder realizar un análisis estadístico y cartográfico que nos permitiera realizar una radiografía de la AS en Cataluña. La creación de esta base de datos partió de la revisión y actualización de fuentes documentales disponibles en la red, ${ }^{5}$ y se ha ido actualizando posteriormente a lo largo de la investigación. La información recogida se clasificó en diversas categorías que después nos facilitaron el análisis y la geolocalización de las experiencias.

La base de datos original constaba de más de un centenar de registros, en los que se incluían entidades que desarrollaban tanto actividades de AS como de jardinería. Esta última categoría se incluyó por el hecho de ser un sector de desarrollo potencial de la AS y desde el cual han surgido algunas iniciativas, pero se excluye del análisis estadístico. La recogida de información inicial se llevó a cabo a partir del envío de 63 cuestionarios a través de Internet, 52 llamadas telefónicas y 22 entrevistas presenciales a una selección de experiencias. Con esta información se obtuvo una primera versión con casi un centenar de registros.

Actualmente la base de datos ha aumentado a 206 registros en 2017 -de los cuales 181 se refieren únicamente a experiencias de AS- debido, en parte, a la ampliación de la información recogida en los ámbitos de actuación de las entidades, considerando no tan solo aquellas que se dedican a la inserción sociolaboral sino también a la terapia, la rehabilitación, la educación y la formación y a los huertos sociales. Los otros 45 registros son de jardinería, ámbito potencial para orientarse a la AS tal como se ha podido comprobar. La actualización de la base de datos se ha

4 Investigación desarrollada en los proyectos de RecerCaixa: "La Agricultura Social en el desarrollo local y la ocupación de colectivos en riesgo de marginación" (2011-2013) y "La Agricultura Social: Análisis económica y evaluación del retorno a la sociedad" (2015-2017).

5 Se consultó el Catàleg de Serveis i Productes de les Empreses d'Inserció (2009), el Catàleg de Centres Especials de Treball (2009) del Departament de Treball de la Generalitat de Catalunya y el Cens de Cooperatives i Societats Laborals (2010), así como las entidades del Tercer Sector Social de Cataluña (http://www.tercersector.cat/) y las entidades trabajan con el colectivo de personas con diversidad funcional (http://www.dincat.cat). 
llevado a cabo mediante una herramienta de investigación colaborativa. Se diseñó un formulario web a través del cual cualquier persona podía proporcionar datos sobre la existencia de una experiencia de AS y permitiéndonos elaborar un mapa colaborativo, a través de la plataforma Instamaps del Institut Cartogràfic i Geològic de Catalunya (ICGC), con el que se pretende reforzar el nexo y las sinergias entre las diferentes entidades y divulgar esta práctica de innovación social y desarrollo local. El mapa está disponible online a través de la plataforma Instamaps, la cual permite consultar la información de la base de datos de manera georeferenciada e interactiva y visualizar mapas temáticos. ${ }^{6}$

\subsection{Análisis cualitativo: cuestionarios y entrevistas en profundidad}

La necesidad de aplicar metodologías cualitativas a nuestra investigación se fundamenta en la voluntad de un análisis profundo sobre el sector, más allá de los datos estadísticos derivados de la base de datos creada, y que no permitiese ahondar en el conocimiento del sector de la AS en Cataluña: las características de las experiencias, la evolución histórica del fenómeno, la implementación territorial de los proyectos y las problemáticas del sector. Se realizaron de manera presencial 19 entrevistas semiestructuradas en profundidad y 3 cuestionarios extensos que los informantes contestaron por vía telemática; en total 22 fuentes de información que corresponden a promotores y/o responsables de iniciativas de AS.

La metodología utilizada para el análisis de los datos recogidos fue, en primer lugar, la transcripción de los archivos de audio y el posterior tratamiento analítico e interpretativo. Como resultado del tratamiento de la información se obtuvo una matriz de datos en la que se mostraban los temas más relevantes referentes a cada una de las experiencias (descripción de los proyectos, trabajo con los colectivos, financiación, evolución y desarrollo del proyecto, valoración) y del sector de la AS (estado y dimensión, descripción y papel de los diferentes agentes, futuro, etc.). ${ }^{7}$

\subsection{DAFO y flujograma}

La recogida de información cualitativa permitió también elaborar un detallado análisis de las debilidades, amenazas, fortalezas y oportunidades (DAFO) de la AS en Cataluña y de los factores que intervienen en el funcionamiento de una entidad. Poder identificar estos elementos y factores de implementación sirvió para realizar una diagnosis exhaustiva del sector y abordar las etapas posteriores, en las que se detectaron, por un lado, los problemas más importantes que dificultaban el desarrollo de la AS en Cataluña, y por el otro, los elementos favorables que lo beneficiaban. En

6 Mapa de las experiencias de AS en Cataluña elaborado a partir de los datos recogidos durante el trabajo de campo. Consultable online: http://bit.ly/2ctcxZT

7 En el informe de resultados, disponible online, aparece una explicación más completa y extensa sobre la metodología utilizada en la investigación:

https://agriculturasocialcat.files.wordpress.com/2014/04/as_memoria_final_ver2.pdf 
este proceso se incorporaron técnicas de trabajo colaborativo entre los miembros del equipo y de las diferentes experiencias, a través del flujograma o mapa de elementos. Esta técnica participativa permite abordar el análisis de un fenómeno complejo a partir del desgranado y clasificación de los diferentes elementos que lo configuran y estableciendo relaciones causales entre ellos que ayudan a establecer un relato interpretativo. Una de las virtudes de esta técnica es que en ella colaboran miembros de un mismo equipo de trabajo, diferentes colectivos o grupos sociales que ayudan a construir una visión conjunta, colectiva y consensuada del objeto de análisis (Riera, 2009). Esta técnica, en nuestro caso, ha tenido como objetivo principal la identificación de un mapa de problemáticas y potencialidades clave y de los principales grupos de interés (stakeholders) de la AS en Cataluña.

\section{La agricultura social en Cataluña: dimensión y características del fenómeno}

Cataluña tiene una superficie de $32108,2 \mathrm{~km}^{2}$ con una población total estimada de 7508106 habitantes en el 2015 (Idescat, 2016). La población activa ocupada en Cataluña es de 3101900 personas (Idescat, 2015a) de las cuales 1,48\%, es decir 46100 personas, están ocupadas en el sector agrario, el cual ha sufrido un claro retroceso en las últimas décadas en términos de ocupación (Idescat, 2015b). La superficie agrícola utilizada (SAU) en Cataluña, incluyendo pastos y tierras cultivadas, ha experimentado un descenso en los últimos 15 años, pasando de 1869203 ha el 1997 a 1125268 ha el 2013 y el nombre de explotaciones también se ha ido reduciendo de 68944 en el año 1997 hasta 59097 al 2013 (Idescat, 2013), siendo las explotaciones pequeñas las que han ido desapareciendo, y las grandes aumentando su tamaño. Sin embargo, frente a este panorama de constante debilitamiento del empleo del sector primario, en Cataluña van apareciendo experiencias que apuestan por la multifuncionalidad agraria, gestando proyectos desde el desarrollo local agroecológico, la innovación social y la elaboración de productos agroalimentarios de calidad, que tienen como denominador común una agricultura más respetuosa y conectada con el territorio. La mayoría de las 161 entidades (en 2017) de AS registradas en la base de datos forman parte de este conjunto de iniciativas que revalorizan la agricultura familiar o de pequeña escala, fomentan el uso respetuoso del territorio y de los recursos a través de la aplicación de criterios de agricultura ecológica y el uso de canales cortos de distribución.

Para descifrar las características de las iniciativas de AS en Cataluña nos hemos basado en el análisis estadístico. En la elaboración de la base de datos recopilamos información referente al ámbito de actuación, los colectivos beneficiarios, la actividad realizada, la forma jurídica, la modalidad de inserción de los colectivos, si aplican o no criterios de producción ecológica y el año de creación del proyecto. A continuación analizaremos este conjunto de experiencias y aportaremos algunas de sus características principales, lo cual nos ayudará a construir una radiografía del sector. 
El ámbito de actuación (Figura 1) de esta práctica social es principalmente la inserción sociolaboral, a través de la que se generan oportunidades para la ocupación de colectivos en riesgo de exclusión social (47\% de los 161 registros). No obstante, aunque en una proporción mucho menor, también aparecen iniciativas que trabajan con la terapia o la rehabilitación (4\%) dirigida, por ejemplo, a las personas con algún tipo de adicción, o bien la educación y la formación (4\%), en proyectos de AS que tienen como objetivo la capacitación de jóvenes que han abandonado los estudios de manera prematura. Por último aparecen los huertos sociales (43\%), un fenómeno creciente en Cataluña como respuesta a las desigualdades y la situación de emergencia social causada por la crisis económica de 2008. Estas son experiencias impulsadas por movimientos sociales, iniciativas ciudadanas y/o la administración local con el objetivo de apoyar a familias y personas individuales frente a situaciones de precariedad.

Cada una de las experiencias de AS centra su trabajo en ciertos colectivos socialmente vulnerables. En su mayoría son personas con discapacidad y/o trastorno mental (35\%). Sin embargo, en años anteriores a la crisis de 2008, la proporción de proyectos centrados en la inserción sociolaboral o la terapia de personas con discapacidad era mucho mayor, experimentado un cierto retroceso en los últimos años. Hay que poner de relieve como esta situación socioeconómica ha ampliado el espectro de la exclusión social, principalmente hacia grupos en situación de privación material o de desempleo, y como esto ha despertado el interés de algunos promotores de iniciativas de AS frente a la necesidad de encontrar soluciones a múltiples situaciones de emergencia social. En este sentido, el $28 \%$ de las iniciativas registradas acogen a personas en situación de pobreza y el $9 \%$ a personas en situación de desempleo, dándoles opciones de empleo a través del trabajo agrario, o bien ofreciéndoles un huerto para la producción de sus propios alimentos. Otro colectivo con una representación importante en las iniciativas es la gente mayor (14\%), debido a la nueva creación y regulación de huertos destinados a personas jubiladas, sobre todo en el entorno metropolitano (Faus, Blanco \& Santos, 2012). También aparecen otros colectivos socialmente vulnerables con menor representación, como jóvenes, niños, personas del ámbito penitenciario, personas inmigradas, mujeres maltratadas o personas sin hogar (14\%).

La mayor parte de estas experiencias se dedican a la agricultura (77\%), especialmente a la horticultura, aunque también está presente el cultivo de la vid, del olivo, el cultivo de setas, entre otros. Otras iniciativas, registradas como AS, se dedican a la transformación agroalimentaria (8\%), como son la transformación de productos lácteos, la elaboración de mermeladas o de cerveza artesana. Los proyectos que desarrollan trabajos forestales y silvicultura representan un $8 \%$ del total. Hay otras entidades que se dedican al sector servicios (5\%) vinculado a la actividad agraria como pueden ser la comercialización a través de circuitos cortos, a la artesanía (1\%) y, por último, actividades de ganadería (1\%). Es importante destacar que los criterios de producción ecológicos 
son aplicados por la mayoría de las experiencias registradas (61\%), lo cual parece ser una fortaleza del sector, entre otras cosas, por la importancia que se da a la mejora de la salud, tanto de las personas beneficiarias como del entorno.

Figura 1. Experiencias de AS en Cataluña según ámbito de actuación (2017)

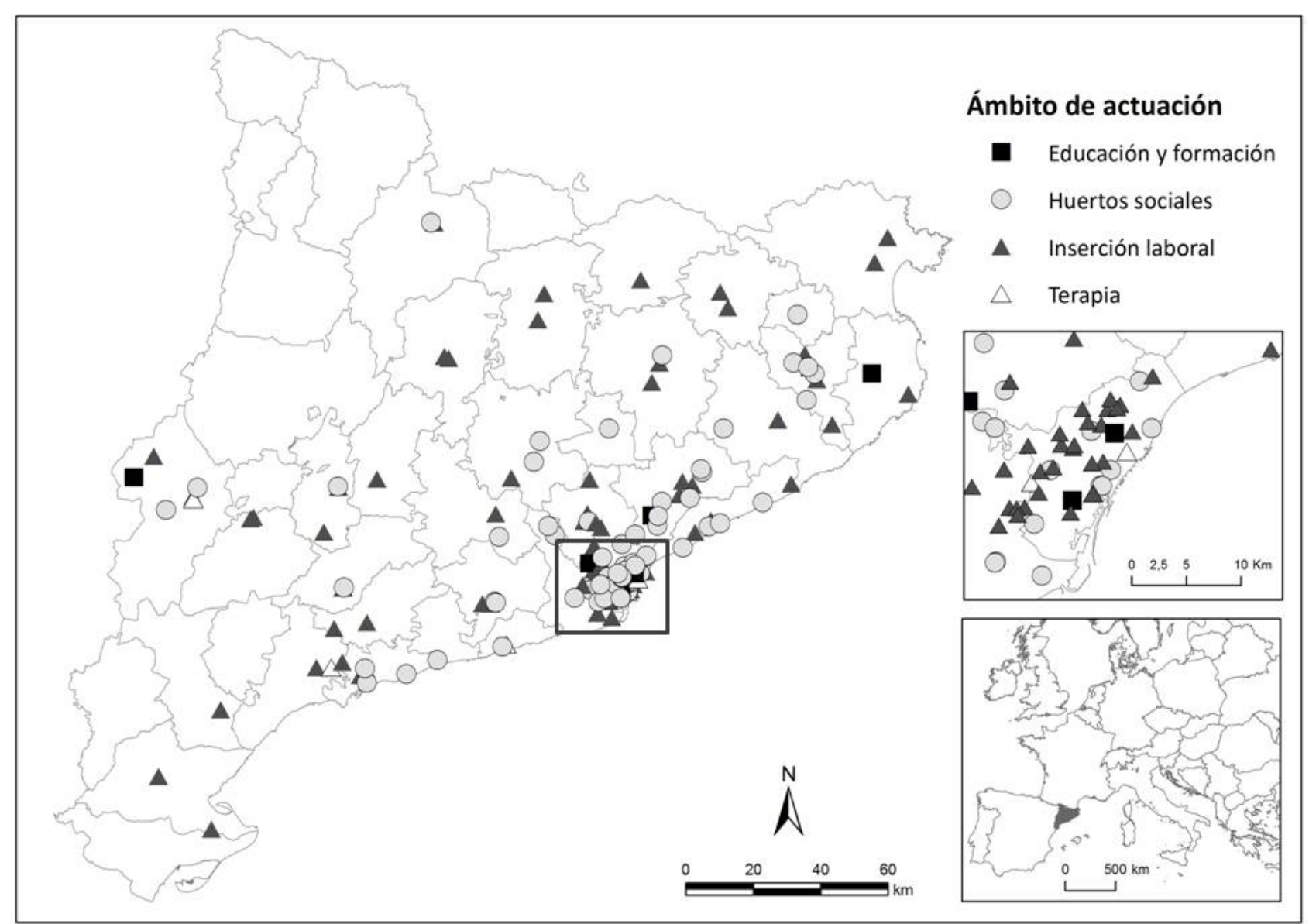

Fuente: elaboración propia a partir de las bases cartográficas de I'Institut Cartogràfic i Geològic de Catalunya (ICGC)

Según el régimen jurídico de las entidades podemos clasificarlas, por un lado, en aquellas instituciones de carácter ciudadano que realizan su actividad sin ánimo de lucro, como son las fundaciones $(14 \%)$ o las asociaciones (22\%), y por la otra las instituciones privadas, algunas de ellas con orientación mercantil, como son las empresas privadas (15\%) y cooperativas (12\%), dentro del TSS (Laville, 2015), con una clara función social y asistencial. También encontramos proyectos de carácter público impulsados desde la Administración (37\%) que en los últimos años está teniendo un papel más proactivo en la implementación y desarrollo de iniciativas de AS en el ámbito local, materializadas principalmente en la creación de huertos sociales. A menudo son proyectos que implican colaboración entre el sector público y privado, con el doble objetivo de fomentar la cohesión social, la economía solidaria y el desarrollo local resiliente (Estela, 2015). 
Las modalidades de inserción de los colectivos que tienen las iniciativas de AS son diversas, estando influenciadas por los colectivos con los que trabajan y responden a la legislación vigente. En primer lugar, los Centros Especiales de Empleo (CEE) $)^{8}$ (43\%), empresas que aseguran el trabajo remunerado a personas con discapacidad, garantizando su integración laboral en un entorno de trabajo protegido. Estos pueden tener un carácter público o privado y están destinados específicamente a las personas con discapacidad ya sea física, intelectual o sensorial. En segundo lugar aparecen las Empresas de Inserción (EI) (12\%), que tienen como objetivo principal la integración sociolaboral de personas en riesgo de exclusión social, ofreciendo una plataforma para reconectarse con el mercado laboral. También hay otras modalidades de inserción, aunque con menor presencia, como los Servicios de Terapia Ocupacional (STO) (2\%) o Centros Ocupacionales (CO), que son una alternativa desde el ámbito de los servicios sociales a la integración de personas con discapacidad intelectual que no se pueden incorporar al sistema de trabajo ordinario.

A parte de las formas de inserción con marcos jurídicos propios, existen otras modalidades, que desde un ámbito informal y de actuación local, tratan de dar solución a situaciones de emergencia social en el contexto local. Este es el caso de diferentes iniciativas sociales (41\%) que surgen en el campo de la AS, las cuales operan desde el prisma del soporte mutuo y la acción colectiva para ofrecer oportunidades a diferentes colectivos socialmente vulnerables, que van desde el huerto para la autoproducción de alimentos a la creación de cooperativas agrícolas para fomentar la autoocupación de personas en situación de desempleo de larga duración. Por último, los Servicios Sociales (17\%) de las diferentes administraciones locales también juegan un papel importante en la inserción de colectivos socialmente vulnerables, principalmente a través de proyectos de carácter público, vinculados principalmente a la creación de los huertos sociales. Aunque no gozan de ninguna figura jurídica específica, como los CEE, las EI o los STO, su trabajo es muy relevante, ya que en muchas ocasiones, aparte de crear sus propios proyectos, colaboran y se coordinan con experiencias de AS en materia de atención social e inserción laboral para mejorar la situación de los colectivos en riesgo de exclusión.

La elaboración y actualización progresiva de la base de datos nos ha permitido analizar la evolución de la AS en Cataluña, estableciendo etapas en su desarrollo. En la década de 1970 aparecen las primeras iniciativas, como es el caso de la Olivera Cooperativa ${ }^{9}$ y de la cooperativa agraria La

8 En Cataluña se denominan Centres Especials de Treball (CET), dependen del Departament d'Empresa i Coneixement y están regulados por el Real Decreto 2273/1985, de 4 de diciembre, y el Real Decreto 1368/1985, de 17 de julio. Más información en http://bit.ly/2cLh6S6

9 L'Olivera Cooperativa fue una de las primeras iniciativas de AS surgidas en Cataluña. Se trata de un proyecto social originado en Vallbona de les Monges (Lleida) en 1974 que aún sigue vigente. Su principal objetivo es ofrecer oportunidades a personas con discapacidad intelectual del y en el entorno rural a través trabajos agrícolas relacionados con el cultivo de la vid, el olivo y la elaboración de vino y aceite y la dotación de servicios de apoyo como un hogar-residencia, un centro ocupacional y profesionales de atención sociosanitaria. Para más información: http://www.olivera.org 
Fageda, ${ }^{10}$ la creación de las cuáles servirían de punto de partida de un sector en crecimiento hasta la actualidad. La dinámica del sector se mantiene en un incremento sostenido desde 1970 hasta mediados de la década de 1990, momento en que se inició un aumento significativo del número de experiencias de AS, coincidiendo con un contexto socioeconómico en que eclosiona el voluntariado y la preocupación por los derechos de colectivos socialmente vulnerables que hasta el momento habían sido invisibilizados (Marbán, Rodríguez, 2006). Además, la recuperación económica, después de la crisis de 1993, permitió dotar a las administraciones públicas de partidas presupuestarias más amplias para dedicarlas a programas de acción social, fomentando la disponibilidad de subvenciones y de ayudas públicas que favorecieron la consolidación del fenómeno. A partir de 2008, la crisis social y económica acaecida provocó la ampliación de los colectivos potenciales de sufrir exclusión social, lo cual hizo saltar las alarmas de las organizaciones del TSS. Durante los años posteriores, la creación de proyectos para dar apoyo a esta situación de emergencia social ha experimentado un aumento sin precedentes que ha hecho aparecer numerosas iniciativas cívicas para dar solución a las necesidades individuales y colectivas de las personas más afectadas por la crisis; la AS no ha quedado al margen de estas dinámica, y durante este período el sector experimenta un incremento exponencial (Figura 2).

Figura 2. Evolución del número de experiencias de AS en Cataluña (1970-2016)

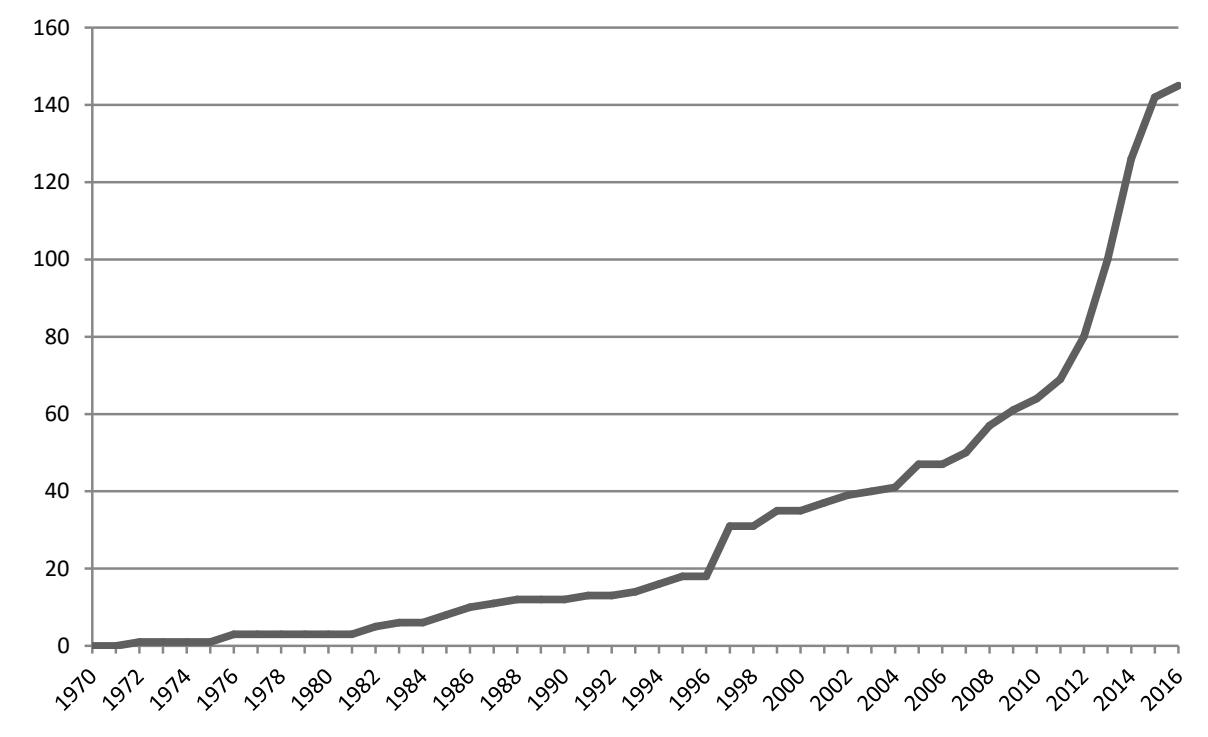

Fuente: elaboración propia

10 La cooperativa La Fageda aparece en 1982 ubicada en el Convento del Carmen (Olot, Girona) con 15 personas que procedían mayoritariamente del psiquiátrico. En 1985 se crea una residencia y el Servicio de Terapia Ocupacional disponiendo de 100 vacas lecheras vendiendo la producción a Nestle-Girona. En 1993 se inicia la producción de 50000 yogures semanales que se venden directamente y a grandes comedores. En 1997 se crea la Fundación y se amplía la gama de producción de lácteos. En 2015 se producen 60 millones de yogurts (5\% cuota de mercado en Cataluña), entre otros productos, y emplean 256 personas (50\% con certificado de discapacidad): http://www.fageda.com/ca 
Por último, y para cerrar este primer apartado sobre la situación de la AS en Cataluña, hemos podido observar como existe una importante concentración territorial de las experiencias de AS, localizándose principalmente en el Área Metropolitana de Barcelona; siendo allí donde encuentran un mayor volumen de población en situaciones vulnerables, mayor disponibilidad de recursos y proximidad a posibles consumidores. Sin embargo, hay una presencia atomizada por todo el territorio, a excepción de las zonas rurales más periféricas como son el Alt Pirineu i Aran y las Terres de l'Ebre.

\section{Discusión}

\subsection{Principales agentes clave de la AS en Cataluña}

La AS vincula de manera inclusiva los servicios sociales y la atención sociosanitaria, con el objetivo de socializar y democratizar los procesos de atención a las personas, extrayéndolos de contextos especializados y llevándolos a ambientes de atención "no formales" como son las asociaciones, cooperativas, empresas y fundaciones. Además, la AS se inscribe en un cambio de paradigma en la asistencia sanitaria y social, dando la oportunidad de realizar tareas y trabajos que contribuyen a potenciar capacidades y habilidades de los usuarios a través de la agricultura y actividades vinculadas. Esta situación también contribuye a lograr una mayor independencia que mejora la situación social de las personas con las que trabaja, en lugar de centrarse en la terapia y la rehabilitación o en las limitaciones de los usuarios (Di lacovo \& O'Connor, 2009).

La naturaleza de este fenómeno reside en la combinación de varios ámbitos de trabajo y disciplinas que tradicionalmente han estado distantes o poco relacionados entre sí como son el sector agrario y el sector social, y que se unen para dar lugar a una nueva manera de abordar conjuntamente la producción de alimentos y la atención sociosanitaria. Esta esencia multidisciplinar provoca que aparezcan múltiples agentes que intervienen en su desarrollo. Las entrevistas nos han permitido detectar qué actores o grupos de interés (stakeholders) son más relevantes y qué papel juegan en la organización del sector en el contexto catalán. Analizando la información obtenida en el trabajo de campo, hemos podido construir un mapa del sector compuesto por 14 agentes clave o grupos de interés.

Las personas usuarias son los sujetos sobre los cuales se centra la actividad y alrededor de los cuales se crean, organizan y estructuran los proyectos. En otros países los usuarios son denominados "clientes", debido a su inclusión en el sistema de atención y su vinculación con los centros proveedores de salud, los cuales ofrecen la AS como tratamiento para la mejora de la salud física y mental (Leck et al., 2014; Dessein et al., 2013). En Cataluña los usuarios no son puntuales, sino que se incorporan al proyecto a medio o largo plazo como parte de la plantilla laboral, cobran 
un salario -en la mayoría de los casos el salario mínimo interprofesional ${ }^{11}$ - y reciben atención integral por parte de técnicos especializados. Otro stakeholder importante, por estar directamente relacionado con las personas usuarias, son las familias. Estas se benefician, en primer lugar, de las mejoras en la situación de sus familiares usuarios, y en segundo lugar, de una cierta tranquilidad y reducción de la dependencia. Cabe destacar que las familias han jugado un papel muy importante en la creación de algunas iniciativas. El anhelo de mejorar la calidad de vida de sus familiares y de ofrecerles oportunidades de ocupación, inserción social y de mejorar su calidad de vida aparece como una pieza clave en el desarrollo de algunos proyectos de AS, siendo en algunos casos los promotores o socios fundadores.

La agricultura es un sector con una gran capacidad terapéutica, mucho más que el sector industrial. [La agricultura] es un trabajo que se realiza principalmente al aire libre, que permite ver todo el proceso, facilita relacionarse con los compañeros y compañeras, potencia el ejercicio físico y la salud, y mejora la psicomotricidad. [AS $1^{12}$ ]

Son muy importante las capacidades terapéuticas que tiene (la agricultura) para las personas que trabajan. A parte, los valores asociados a la producción agraria ecológica hace que terapéuticamente evolucionen más favorablemente, y a la vez sea una manera de mostrar que otro tipo de alimentación y de territorio es posible y necesario. [AS4]

Los agentes promotores, que a veces coinciden con las familias, también son un stakeholder relevante. Son personas o entidades que detectan la necesidad, aplican los recursos, desarrollan el proyecto y los llevan a cabo. La tipología de estos actores es heterogénea y en muchos casos crean estructuras ad hoc, en función de la coyuntura particular de cada iniciativa, para dar entidad legal al proyecto y empezar su andadura en el campo de la AS. Uno de los modelos más recurrentes son las asociaciones o fundaciones del TSS, con una amplia experiencia en el servicio asistencial e inserción laboral de personas con discapacidad intelectual, que actúan como entidad matriz, creando proyectos de AS con la voluntad de diversificar sus actividades, ofrecer nuevos servicios y oportunidades para los colectivos con los que trabajan.

En la gestión de los proyectos también tienen un papel relevante los trabajadores especializados, los cuales pueden ser personal de atención sanitaria, social o del ámbito agrario. La mayoría de proyectos cuentan con unidades de apoyo, formadas por personal sociosanitario especializado como técnicos de inserción, educadores sociales, psicólogos y/o asistentes sociales, que ofrecen

11 El Salario Mínimo Interprofesional (SMI) para el 2015 era de 648,60€, tal y como establece el Real Decreto 1106/2014, de 26 de Diciembre, publicado en el Boletín Oficial del Estado (BOE, 27 de Diciembre de 2014, número 313, Sec. I, pp. 105840).

12 A cada una de las personas entrevistadas se les ha asignado un código para mantener la confidencialidad de los informantes. Los códigos utilizados son "ASn", para las personas entrevistadas vinculadas con algún proyecto de $\mathrm{AS}, \mathrm{e}$ "IC $n^{\prime}$, para las personas entrevistadas como informantes clave. 
atención integral a los colectivos de usuarios, diseñando itinerarios de inserción laboral individualizados, planes de inclusión social y acompañamiento psicológico. Asimismo, los proyectos cuentan con técnicos agrarios o agroalimentarios que se encargan de planificar los cultivos, las características de los productos elaborados, el sistema de producción y la estrategia de comercialización. Pese a ser una pieza clave en el desarrollo de los proyectos de AS, algunos no cuentan con este tipo personal especializado en la producción, ya que el origen del proyecto se ha iniciado en el seno de una entidad de atención social y no dispone de estos recursos.

La ley ya contempla que a las personas que trabajan se les debe ofrecer un apoyo y un seguimiento para realizar las diferentes tareas relacionadas con la actividad empresarial que se desarrolla. Nosotros tenemos unos monitores en el centro que apoyan y supervisan el trabajo que hacen los diferentes trabajadores, un equipo de psicólogos y trabajadores sociales que desde las áreas de cada cual, trabajan para darle un apoyo integral en cada persona. Son totalmente necesarios. [AS5]

Muchas empresas pequeñas formalizadas como CET o El no podemos tener personas contratadas en plantilla que hagan estas tareas de apoyo, porque los gastos serían muy grandes. En nuestro caso aprovechamos los recursos humanos de [entidad del TSS], que tiene un grupo de personas formadas que desarrollan esta tarea. [AS4]

También cabe destacar que la mayoría de iniciativas se nutren del trabajo de personas voluntarias, las cuáles, por afinidad, satisfacción y voluntad de contribuir a un proyecto social, dedican su tiempo y sus conocimientos a esta actividad. El perfil mayoritario son personas jóvenes (principalmente estudiantes), personas en situación de desempleo y personas jubiladas que tienen una dedicación horaria aproximada entre 5 y 10 horas semanales durante un periodo de implicación de uno a dos años. Hemos podido observar que esta característica de la AS aparece de manera recurrente en otros países como un pilar fundamental en el desarrollo de esta actividad (Hine et al., 2008; Fazzi, 2011; Di lacovo et al., 2014).

La AS ofrece servicios en diferentes ámbitos dedicados a ofrecer oportunidades de inclusión de personas en riesgo de exclusión social, pero también produce y elabora productos agroalimentarios que son consumidos por personas con unas pautas de consumo determinadas. La calidad, lo ecológico, lo social y la proximidad son algunos de los elementos que llevan asociados los productos de las experiencias de AS y que encuentran en ciertos consumidores su segmento de mercado. Los proveedores, por su parte, son aquellos que aportan las materias primas o recursos necesarios que permiten a las entidades llevar a cabo su actividad. No todos los proveedores de las entidades de AS tienen la misma importancia, ya que existen ciertos proveedores clave, como los de insumos ecológicos, que tienen un papel destacado en el avance de los proyectos, implicándose en el proyecto. 
La Administración Pública ${ }^{13}$ juega un papel dual en el sector de la AS en Cataluña, combinando el rol de facilitador "pasivo" y de beneficiario. Por una parte, ofrece subvenciones para mantener el trabajo protegido, ayudas con las que las experiencias pagan las retribuciones a los trabajadores, o bonificaciones para la contribución a la Seguridad Social con el fin de establecer un sistema de incentivos para la contratación de colectivos en riesgo de exclusión social. Dichas ayudas varían en función de la naturaleza jurídica de la empresa y de la situación legal de las personas usuarias. Por otra parte, la Administración Pública aparece como un stakeholder que se beneficia de la actividad de los proyectos de AS. El trabajo que realizan las entidades repercute directamente en las arcas públicas del Estado y de la Generalitat de Catalunya, haciendo más eficientes las inversiones. Según han manifestado las personas entrevistadas se produce un importante ahorro presupuestario, ya que la mejoras que se dan provocan un descenso en la frecuentación al sistema de salud pública, minimiza el pago de subsidios, como prestaciones por desempleo y rendas mínima de inserción (RMI), ${ }^{14}$ y economiza esfuerzos y recursos en programas de cohesión social, dotación de servicios sociosanitarios (principalmente en áreas rurales) e inserción laboral.

[La Administración] se tendría que sensibilizar más, es verdad que hay trabajos que es difícil cubrirlos con estas personas, pero la responsabilidad tendría que ser más compartida, entre los centros y la administración. La administración lo tendría que tener como una prioridad, puesto que si ya es difícil por todo el mundo, para las personas en riesgo (de exclusión) todavía lo es más. A parte, este tipo de experiencias suponen un ahorro importante en el gasto público. A nivel político no se dan cuenta. [...] Nosotros tuvimos que hacer un ERE. Tuvimos que despedir a 20 personas con trastorno mental. Estas personas ya les cuesta de por sí encontrar trabajo en centros especializados, así que o la administración les das un incentivo laboral o tienen las de perder, porque la verdad es que las empresas convencionales no incorporan esta gente a las plantillas. [AS9]

Tener una persona con discapacidad o enfermedad mental parada es mucho más caro, tanto a nivel humano como nivel económico, que tenerla ocupada en una tarea en la que se sienta útil y se pueda relacionar con otras personas. Una persona cerrada en

13 Las subvenciones y ayudas para mantener las plazas de trabajo protegido en los CET y El o las plazas en los centros ocupacionales a las personas usuarias las ofrece el Departament de Treball, Afers Socials i Famílies de la Generalitat de Catalunya, que tienen conferidas las competencias en materia de ocupación y bienestar social. Por su parte, las bonificaciones en las contribuciones a la Seguridad Social las dictamina el Ministerio de Empleo y Seguridad Social del Gobierno de España.

14 La Renda Mínima d'Inserció (RMI) es una acción de solidaridad de carácter universal que tiene como finalidad el desarrollo coordinado de las acciones destinadas a ayudar a las personas que no disponen de los medios económicos suficientes para atender las necesidades esenciales de la vida cotidiana, mientras se las prepara para su inserción o reinserción social y laboral. 
casa, sin relacionarse, dependiendo del sistema sanitario, sale muy "cara" por el gobierno, aunque no se den cuenta, o no quieran darse cuenta. [AS1]

El acceso a los recursos, tanto físicos como financieros, es uno de los factores clave para el éxito de las iniciativas de AS. Es por eso que las personas y entidades con tierras en propiedad representan un stakeholder importante en el impulso y difusión de la AS en Cataluña, ya que uno de los principales obstáculos con los que se encuentran es la dificultad del acceso a la tierra, debido a la falta de regulación y especulación con el precio de la tierra. En algunos casos, la administración local cede temporalmente o arrienda tierras de titularidad pública a proyectos de AS, dando solución puntualmente este problema. Sucede lo mismo con los agentes financiadores; muy importantes en las primeras etapas de los proyectos. El origen de las inversiones y el financiamiento es bastante heterogéneo y varía en función de cada proyecto y de los vínculos particulares que cada entidad tenga con instituciones y organismos con capacidad financiadora, pero la mayoría buscan apoyo económico en las ayudas de la administración pública, cada vez más escasas, y de organizaciones privadas que estén en concordancia con sus valores y objetivos. Sin embargo, hemos detectado que hay "entidades madre" (fundaciones, asociaciones) que garantizan el financiamiento durante los primeros años de los proyectos y subsanan posibles pérdidas económicas durante el desarrollo de la actividad.

Ahora (en referencia a los efectos de la crisis económica) es difícil salir adelante sin subvenciones, pero también lo era hace treinta años atrás y la gente bien se ponía a trabajar y salía adelante. Compramos algunas tierras con la herencia de alguno de los miembros de [la entidad]. íbamos tirando con lo que podíamos. Poco a poco, el sector se va estructurando y nos empiezan a llegar ayudas de Benestar Social [Departamento de la Generalitat de Catalunya] y empezamos a presentarnos a las convocatorias del Fondo Social Europeo, a través del programa Leader, y porque nuestro producto encajaba muy bien con las líneas de la política europea. También todo lo que íbamos ganando lo íbamos reinvirtiendo en la entidad, y no con sueldos, que los manteníamos bajos porque ya nos permitían vivir. Preferíamos crear puestos de trabajo, mejorar instalaciones, etc. La prioridad era consolidar el proyecto y el equipo. [AS6]

Nosotros tenemos que buscar la viabilidad pura y dura, ya que vivimos sin subvenciones. Hemos tenido que crear una estructura de empresa convencional, y eso también es innovador en el financiamiento de las empresas sociales. Esto tiene un riesgo, quiere decir que si no salen los números, pues tenemos que ir jugando con los personal contratado y los gastos, como cualquier empresa, pero con la diferencia que trabajamos con personas socialmente vulnerables. [AS7] 
Algunas de les entidades entrevistadas han sido creadas (o consolidadas) a través del apoyo recibido por parte de agentes emprendedores. Este stakeholder está formado por actores (organismos públicos, privados y mixtos), que fomentan el emprendimiento y el desarrollo de proyectos de economía social a través de programas específicos de formación, asesoramiento y acompañamiento. En Cataluña, el gobierno lleva años desarrollando con éxito el programa @EmprenSocial ${ }^{15}$ de emprendimiento social, por el cual han pasado algunos de los proyectos de AS entrevistados, y que ofrece un plan individualizado a las empresas sociales, ayudándolas a definir su proyecto empresarial y compatibilizar su misión social con la viabilidad económica.

A inicios de la década de 2010, el sector de la AS se componía de experiencias con un elevado grado de atomización, entre las cuales no existía vínculos institucionalizados que fomentasen la colaboración o cooperación entre ellas y que ayudasen a consolidar el sector (Guirado et al., 2013). Únicamente había una red de carácter privado, la Xarxa AgroSocial, ${ }^{16}$ creada desde una fundación privada vinculada a una entidad financiera, que daba apoyo a determinados proyectos. Con el tiempo han ido apareciendo nuevas redes de soporte, sobre todo en el ámbito de la comercialización conjunta, como es el caso de 2147 Mans $^{17}$ o Vogadors, que agrupan diferentes iniciativas del sector. Las sinergias entre las diferentes iniciativas cada vez son más fuertes, sin embargo aún no existe una red que gestione toda la AS a escala regional y que integre todos aquellos proyectos que trabajan en este ámbito de innovación social, los coordine, los represente delante de instancias públicas y privadas, les dé recursos de apoyo y asesoramiento y divulgue el papel de la AS entre la sociedad.

Existen muchas experiencias, pero no es comparable por ejemplo con Francia o Italia. La diferencia entre Cataluña e Italia, es que allí hay una reflexión teórica muy potente que aquí no hay. Allí se ha pensado mucho más sobre el tema. [...] [En Cataluña] estamos todavía en una fase de pequeñas explotaciones que quieren incorporar el elemento social a sus proyectos, pero faltaría un poco la reflexión de territorio, de conexión con el desarrollo rural, con la producción ecológica, etc. [...] Falta esta reflexión más integrada entre alimentación, agricultura, territorio, modelo de desarrollo y economía social. [AS6]

15 @EmprenSocial (http://www.emprenedoriasocial.cat) es un programa marco, impulsado por el Departament d'Empresa i Ocupació de la Generalitat de Catalunya, que tiene como objetivos fortalecer el ecosistema del emprendimiento social con la participación de las entidades e instituciones socias y ampliar la visibilidad y la presencia de la emprendimiento social al conjunto de la sociedad catalana.

16 Xarxa AgroSocial de la Fundación Catalunya-La Pedrera es una plataforma que da soporte a la creación y consolidación de empresas en el ámbito de la economía social, dedicadas a la actividad relacionada con la agricultura y/o el medio ambiente. Actualmente cuenta con 23 miembros, la mayoría de los cuales son proyectos de AS. Para más información: http://www.fundaciocatalunya-lapedrera.com/es/content/red-agrosocial

17 Para más información: http://www.2147mans.coop/ 
Trabajamos de manera aislada y hay pocas experiencias de trabajo conjunto y creación de redes. [...] Sería muy positivo y necesario la creación de sinergias y redes sólidas de relación y cooperación con otras iniciativas, que nos permitiera ser más competitivos, más fuertes ante la administración y la empresa privada y ser más visibles en el mercado. [AS1]

Las iniciativas de AS se tienen que juntar para ser fuertes en términos comerciales, porque el poder que tú tienes de venta produciendo pequeñas cantidades o uniéndote ofreciendo un volumen competitivo en términos de mercado es muy diferente. [AS7]

Por último, la AS aparece como una clara muestra de innovación social, estrechamente relacionada con la acción colectiva, la cohesión y la interacción activa de la personas con los recursos locales, generando importantes impactos en la comunidad local y el entorno territorial. La agricultura, y por extensión el resto de subsectores agrarios, precisa de un vínculo con el territorio que contribuye a hacer de esta práctica un elemento clave en el desarrollo local de territorios rurales y periurbanos, esta actividad vertebra la gestación de proyectos innovadores, en la que se crean nuevas oportunidades de ocupación -tanto para personas en riesgo de exclusión como para la población en general-, se tejen redes de cooperación, un hecho que ayuda a fijar población al territorio y favorece la ampliación de la dotación de servicios en estas áreas, en las que a menudo existe un déficit en el sistema asistencial de los colectivos más vulnerables. En el aspecto ambiental, la AS contribuye a un mantenimiento y buena gestión de los mosaicos agrícolas y forestales, así como a la conservación de la biodiversidad y la prevención de incendios, principalmente en el ámbito periurbano, ya que los proyectos se instalan sobre todo en terrenos abandonados de las zonas de interfaz urbano-forestal, con un elevado valor paisajístico y ambiental (Badia \& Valldeperas, 2015).

\subsection{Diagnosis del sector}

El tratamiento de la información derivado de las entrevistas a los agentes clave del sector de la AS en Cataluña y el trabajo de análisis realizado posteriormente nos ha permitido abordar la diagnosis del sector a través de la definición de sus características internas (fortalezas y debilidades) y los elementos relacionados con el entorno que afectan directa o indirectamente al sector (amenazas y oportunidades). A continuación describiremos algunos de los aspectos más relevantes de este análisis DAFO y apuntaremos algunos de los retos que se les plantean al sector.

Las fortalezas del sector de la AS en Cataluña están estructuradas en cuatro grandes grupos: la agricultura y el sector agroalimentario como sectores estratégicos, la vinculación de los proyectos al territorio y la contribución al desarrollo local, la innovación social y los beneficios que esta actividad ejerce sobre las personas usuarias.

El sector agrario y agroalimentario se encuentra actualmente en una situación ventajosa. La producción de alimentos vive un momento de expansión en Cataluña; configurándose como un 
sector estratégico que pretende cubrir los diferentes segmentos del mercado, cada vez más preocupados por el producto de calidad, cercano, con criterios éticos y de respeto por el medio ambiente. La AS defiende de manera casi generalizada un modelo agrario basado en productos locales, de temporada, y en muchos casos, también con criterios ecológicos, y que a su vez recupera el valor del campesinado, asignando nuevos roles a la actividad agraria más allá de su vertiente meramente productiva. Además, la implementación de criterios sociales, inherentes a la práctica de la AS, dota de un valor añadido a la actividad agraria y a los productos que se ofrecen, siendo una muestra clara de multifuncionalidad rural.

Por otra parte, los vínculos que tienen las experiencias con el territorio aparecen como una fortaleza destacable del sector, contribuyendo de manera positiva en diferentes ámbitos como el desarrollo local, la cohesión social, la gestión de les recursos y la conservación del medio. El arraigo territorial de las experiencias las convierte en importantes agentes dinamizadores en el entorno rural y periurbano, ayudando a la creación de servicios y redes de apoyo para las personas en riesgo de exclusión social, que a veces son escasos. También hemos podido comprobar cómo la creación de iniciativas ayuda a establecer redes de cooperación entre diferentes agentes de un mismo territorio, así como sinergias con otras iniciativas y nuevas relaciones entre productores y consumidores que modifican las redes agroalimentarias hegemónicas.

La AS ofrece también servicios de atención social y terapéutica a través del trabajo agrario. La complementariedad que se consigue a través de la unión de la esfera de lo social y lo agrario le otorga a esta práctica un carácter claramente innovador, ya que genera alternativas económicas al servicio de las personas y que promueven valores como la solidaridad, el cooperativismo y la mutualidad. En este sentido, una de las fortalezas de la AS es la iniciativa ciudadana, la voluntad de crear proyectos que den solución a problemas colectivos y que suplan las carencias del sistema de bienestar. Por otra parte, una de las fortalezas clave, y en torno a la que orbita toda la práctica de la AS, es la mejora en la vida de las personas beneficiarias. Las experiencias han demostrado como a través del trabajo agrario los usuarios experimentan importantes beneficios, sobre todo relacionados con la salud física y emocional, la autonomía en la vida cotidiana, la mejora de las relaciones interpersonales y la adquisición de nuevas habilidades.

El sector de la AS en Cataluña también presenta una serie de debilidades, que según lo que hemos podido observar a lo largo del análisis, están relacionadas con la propia dinámica y situación del sector y la gestión de las experiencias. La mayoría de proyectos de AS son de reciente creación y el grado de desarrollo de este fenómeno en Cataluña no cuenta con una larga evolución. Según Di lacovo (2010) existen diferentes estadios de desarrollo de la AS, desde una etapa inicial, donde las experiencias son escasas y tienen un bajo reconocimiento social, hasta una etapa de nueva consciencia, en la que la AS forma parte de los servicios a la comunidad con un reconocimiento social e institucional pleno y un marco normativo propio. En el caso de Cataluña, consideramos que 
el sector está pasando de una etapa inicial, o de novedad, a una etapa de impulso, en la que cada vez hay más experiencias y empiezan a crearse redes de colaboración y programas de fomento de la AS en el ámbito local. Sin embargo, el establecimiento de estas sinergias no es suficiente para la cohesión del sector y algunas experiencias aún se encuentran aisladas, con poca relación con otros proyectos y con un escaso apoyo por parte de la administración, dando lugar a un sector atomizado y con poco reconocimiento institucional y escasa visibilidad social.

Otra debilidad de las experiencias de AS es que los rendimientos productivos son más bajos que los de la empresa convencional. Esta diferencia se hace todavía más evidente si lo comparamos con las empresas del sector agrario, hecho que provoca que el agravio comparativo sea más grande y que disminuya la competitividad de las iniciativas de AS en el mercado. Estos bajos rendimientos son causados por la dificultad de adecuar las tareas de la explotación o el obrador a las especificidades de los diferentes colectivos de usuarios. Sin embargo, se acepta socialmente un precio ligeramente más elevado (10-20\%).

En tercer lugar, las oportunidades están condicionadas por la coyuntura económica y la capacidad inclusiva del sector. El contexto socioeconómico de crisis ha sido valorado por las personas entrevistadas como un escenario propicio para innovar y emprender. Algunos de estos emprendedores lo hacen en el ámbito de la economía social, dotando a las iniciativas de unos valores más justos y solidarios. El desarrollo del sector de la AS en Cataluña se puede asociar al surgimiento de nuevos modelos económicos y financieros como alternativa al modelo económico actual, así como a la defensa de una nueva ética centrada en la justicia territorial, la justicia social, la igualdad y el empoderamiento de los sectores más vulnerables de la sociedad. Estos cambios van acompañados de un proceso de innovación social basado en la inclusión, la cohesión, la iniciativa cívica y la ayuda mutua.

La mayor visibilidad de la AS también se percibe como una oportunidad para su desarrollo futuro. En los últimos años ha habido un creciente reconocimiento del fenómeno como vía de acción política para solucionar ciertas situaciones de emergencia social, pero también como estrategia de desarrollo local, de inserción sociolaboral, de mejora de la salud, etcétera. Estas circunstancias han provocado que la AS esté cada vez más presente en la agenda de las administraciones públicas.

Por último, encontramos las amenazas a las que se enfrenta el sector. Estas están relacionadas con el contexto socioeconómico -el cual aparece a la vez también como oportunidad_, el escaso reconocimiento social y la ausencia de dinamización del sector por parte de las instituciones. En el contexto socioeconómico actual, el estado de bienestar se ha ido debilitando progresivamente, perdiendo capacidad de atender las demandas de una población ampliamente afectada por la crisis y los recortes en materia de salud, educación y servicios sociales, entre otros ámbitos. Según afirman las personas entrevistadas, la administración se ve a menudo incapaz de liquidar 
subvenciones pendientes para mantener las plazas de trabajo protegido en los CET y las El, aparte de vetar la creación de nuevos centros de inserción sociolaboral debido a las limitaciones presupuestarias, en un momento en el que la situación social más los reclama.

A todo esto, se añade la escasa atención que recibe la AS por parte de la sociedad y la administración. A pesar de que hay un despertar en la conciencia de la sociedad en relación a la economía social, todavía falta que estas alternativas económicas representen una parte importante y visible de la actividad económica de Cataluña. La AS, en particular, todavía es un sector desconocido por la gran mayoría de la sociedad; y las instituciones políticas, si bien algunas reconocen la existencia del fenómeno, lo hacen de forma muy tímida y sin acompañarse de ningún programa que incentive su impulso ni de un marco jurídico que fomente la creación de nuevos proyectos. En cambio, hay un fuerte convencimiento de la utilidad de los proyectos de AS en la UE que está favoreciendo políticas estratégicas para su desarrollo (Willems, 2013).

\section{Tabla 1. Resultados del flujograma: factores de desarrollo y ejes de trabajo}

FACTORES FAVORABLES

\section{FACTORES LIMITADORES}

\begin{tabular}{ll}
\hline Cambio de paradigma agrosocial & Contexto de crisis \\
Cohesión social y dinamización territorial & Escasa valoración económica y social de la AS \\
\hline Fomento de la economía social & Sector emergente con escaso reconocimiento social \\
Nuevo rol de la agricultura & Condicionantes legales y administrativos \\
Nuevos modelos de gestión y organización & Minimización de los recursos \\
Cooperación incipiente & $\begin{array}{l}\text { Carencia de vinculación y coordinación entre } \\
\text { agentes }\end{array}$ \\
$\begin{array}{l}\text { Atención integral y beneficios para los } \\
\text { colectivos en riesgo de exclusión }\end{array}$ & Sector heterogéneo y poco cohesionado \\
& Limitaciones en la ocupación de algunos colectivos \\
\hline
\end{tabular}

Fuente: elaboración propia

Una vez realizado el análisis DAFO, se utilizó el flujograma, una herramienta de planificación estratégica participativa (Riera, 2009) que nos permitió identificar los principales elementos clave y, finalmente, esbozar algunas posibles acciones que favorecieran el desarrollo de la AS. En el flujograma se consideraron los factores favorables (fortalezas y oportunidades) y los factores limitadores (debilidades y amenazas) de forma agrupada. Los elementos de cada grupo estaban numerados y eran hasta un total de 52, después de aplicar la metodología del flujograma, se llegó a 7 categorías favorables que agrupaban 29 factores, y a 8 categorías limitadoras que agrupan 23 factores (tabla 1, columnas segunda y tercera). Estas categorías clasificadas en 5 ejes de trabajo (primera columna de las tablas I y II) nos permiten jerarquizar el análisis y las recomendaciones. ${ }^{18}$

18 La complejidad de esta metodología y su extensión nos impide desarrollarla en este artículo y, por ello, se debe consultar la memoria de resultados de la investigación (pp. 187-219) en la web: https://agriculturasocialcat.wordpress.com/resultats/ 


\section{Tabla 2. Acciones estratégicas recomendadas para impulsar la AS en Cataluña}

EJE DE TRABAJO

\section{ACCIONES ESTRATÉGICAS}

\begin{tabular}{|c|c|}
\hline 1. Contexto social y económico & $\begin{array}{l}\text { 1.1. Programa de fomento de la AS como un sector de la economía social } \\
\text { 1.2. Difusión y visibilidad de la AS }\end{array}$ \\
\hline $\begin{array}{l}\text { 2. Consolidación del sector e } \\
\text { impulso de la AS }\end{array}$ & $\begin{array}{l}\text { 2.1. Elaboración de un marco jurílico/normativo propio } \\
\text { 2.2. Inclusión de la AS en las políticas públicas } \\
\text { 2.3. Evaluación del retorno social (SROI) }\end{array}$ \\
\hline $\begin{array}{l}\text { 3. Dotación de recursos e } \\
\text { infraestructuras }\end{array}$ & $\begin{array}{l}\text { 3.1. Facilitar el acceso a vías alternativas de financiación } \\
\text { 3.2. Reserva de tierras para la AS }\end{array}$ \\
\hline 4. Cohesión interna del sector & 4.1. Creación de una plataforma o red de AS \\
\hline 5. Ampliación y extensión de la AS & $\begin{array}{l}\text { 5.1. Explorar nuevos campos de desarrollo de las experiencias de AS } \\
\text { 5.2. Fomento de la AS como una estrategia de desarrollo local } \\
\text { 5.3. Programa de formación específica para los profesionales del sector }\end{array}$ \\
\hline
\end{tabular}

Fuente: elaboración propia

Los factores nos muestran algunos de los elementos de diagnosis determinantes en cada eje de trabajo. Este análisis nos ha llevado a hacer una primera aproximación a posibles acciones estratégicas que se podrían llevar a cabo para impulsar la AS en Cataluña y paliar las debilidades o amenazas detectadas, aprovechando el impulso positivo de las fortalezas u oportunidades identificadas. Para cada acción estratégica, se ha especificado el eje de trabajo a la que corresponde y el ámbito de actuación en que se podría desarrollar. En el anexo del informe de resultados del proyecto se amplía la información con una ficha para cada una de las acciones en la que aparece la justificación, la descripción de la acción, los objetivos, los agentes implicados y las observaciones y restricciones que comporta (Tulla et al., 2014, pp. 209-219). En el presente artículo, únicamente enumeramos las acciones, enmarcándolas en el eje de trabajo correspondiente.

\section{Consideraciones finales}

La práctica de la AS ha crecido considerablemente en los últimos años en Europa, sin embargo la dimensión y la evolución de esta práctica se percibe como un fenómeno heterogéneo en el conjunto de los países europeos, ya que existen importantes diferencias territoriales y diversos estadios de desarrollo del fenómeno. En el caso de Cataluña la AS se encuentra en una fase inicial de desarrollo, es un sector que ha experimentado un crecimiento considerable en las últimas décadas. A diferencia de lo que pasa en otros países como por ejemplo Italia, en Cataluña la participación de los entes públicos es más bien baja. Esta se resume a experiencias concretas impulsadas por la administración local, como por ejemplo los huertos sociales o comunitarios (Pomar \& Tendero, 2015). También existen las subvenciones o ayudas que tienen por objetivo respaldar los proyectos que desarrollan acción social o de promoción de la agricultura ecológica y que al mismo tiempo favorecen el desarrollo rural mediante la AS. Sin embargo, es un fenómeno en progresiva expansión donde cada vez van apareciendo nuevas experiencias y redes que ayudan 
a consolidar esta práctica. El crecimiento del fenómeno junto con el reconocimiento cada vez mayor por parte de la sociedad y de las administraciones públicas repercute en mejorar la identidad colectiva de las entidades, permitiendo visibilizar los beneficios que estas actividades aportan a la sociedad.

La AS contribuye a empoderar y generar oportunidades de ocupación para personas en riesgo de exclusión social, mejorando la autonomía, el bienestar personal, y la inclusión social de los colectivos con mayor fragilidad social. El trabajo en las entidades de AS genera, en el ámbito personal, beneficios en la salud física y mental de las personas, y una mejora en la salud emocional, contribuyendo a aumentar la autoestima y la confianza en sí mismos. Son iniciativas sociales y solidarias por la prioridad que dan a la inserción laboral y la mejora en la salud mental y física de los usuarios frente a la ganancia económica de la entidad. En el ámbito del desarrollo territorial, la AS favorece la creación de servicios de atención social y sanitaria en áreas rurales periféricas donde se implantan las experiencias de AS. Esto permite ampliar la dotación de servicios y la cobertura territorial, pudiéndose reducir la exclusión social y los desequilibrios territoriales.

La fragmentación del estado del bienestar desde un punto de vista de crisis económica y de políticas de austeridad impuestas por la UE en los países del sur de Europa junto con la situación actual de emergencia social frente a las carencias explican la reciente aparición de iniciativas de nueva creación de AS en Cataluña (Laparra \& Pérez, 2012, Pavolini et al., 2015; Guillén et al., 2015). Así pues, la AS se constituye como un elemento catalizador de alternativas económicas, debido a que son actividades que aportan valores de transformación social y de justicia, las cuales ponen por delante las necesidades de los colectivos más vulnerables. Por ello, la AS aparece como resultado de demandas sociales comunas y representa una herramienta eficaz para construir alternativas económicas con valor social añadido, al mismo tiempo que se revaloriza la agricultura.

De este modo, la AS aparece como práctica de innovación social, relacionada con la mutualidad, la acción colectiva, la cohesión y la interacción activa de la personas a nivel particular, familiar y comunitario con los recursos locales y con poca interacción del estado. Estas son características que definen el sistema de bienestar mediterráneo donde la sociedad civil ocupa un lugar muy importante (Naldini, 2003). La AS, como actividad de la economía social, solidaria y cooperativa, obvia los planteamientos únicamente económicos y se enfoca en cómo utilizar los recursos locales para un desarrollo económico resiliente, que atienda a las necesidades sociales, aporte un mayor bienestar individual y colectivo y fomente la sostenibilidad económica, ambiental, social y territorial (Walker \& Salt, 2006). En este sentido, esta práctica subyace como una estrategia para generar economías locales de nueva creación, más centradas en las especificidades, las potencialidades y las oportunidades de cada territorio, que en las tendencias y la conectividad con la economía global (Estela, 2015). Es por eso que la AS se presenta con diferentes formas en distintos 
territorios, heterogeneidad resultado de las estrategias de resiliencia de cada territorio para dar respuesta a sus retos y adaptarse a los cambios (Tulla et al., 2017).

En Cataluña un gran número de experiencias de AS trabajan en la implementación de criterios ecológicos y con planteamientos agroecológicos para la producción agraria aproximándose a nuevas lógicas en la práctica agraria alejadas de las prácticas agroindustriales hegemónicas. De este modo se forjan redes de cooperación entre consumidores y productores, elaborando proyectos fuertemente vinculados con el territorio y la comunidad, aportando significado a las múltiples funciones del trabajo agrario y ofreciendo productos de calidad, ambientalmente sostenibles y socialmente justos.

Por último, concluimos que la AS surge como una práctica con un retorno social importante, mediante la cual las inversiones públicas y privadas aportan un impacto importante en la sociedad, tanto en la esfera económica, como social y ambiental. Añadimos que la AS hace más eficiente la gestión de los recursos públicos (Guirado et al., 2017), debido a que esta rentabiliza las inversiones realizadas en los proyectos con relevantes cambios sociales y ambientales, y además porque optimiza el gasto público en materia de servicios sociales, de salud, de gestión del territorio y de mejora ambiental a raíz de los cambios generados por la AS.

Agradecimientos: Se ha realizado esta investigación con los proyectos 2011ACUP023 y 2014ACUP029 (RecerCaixa) y el proyecto CSO 2012-31979 (MEC) que ha cofinanciado. El equipo de investigación en Agricultura Social de la UAB (ERAS) lo forman: Anna Badia, Àngel Cebollada, Carles Guirado, Antoni F. Tulla (IP), Natàlia Valldeperas y Ana Vera, del Departament de Geografia, Josep Lluís Espluga, del Departament de Sociologia, y las profesionales autónomas Camile Evard e Imma Pallarès-Blanch (ingenieras agrónomas) y Laia Sendra (ADE), antes en la Fundació Centre de Desenvolupament Rural Integrat de Catalunya (CEDRICAT).

Declaración responsable: Las/os autoras/es declaran que no existe ningún conflicto de interés en relación a la publicación de este artículo. Las tareas se han distribuido de la siguiente manera: el artículo ha sido coordinado por C. Guirado. Las/os cuatro autoras/es han participado en la revisión bibliográfica, el análisis DAFO y la redacción del artículo. La elaboración de la base de datos estuvo a cargo de C. Guirado y N. Valldeperas, incorporándose A.F. Tulla en el trabajo de campo y el análisis cualitativo, y A. Vera en el análisis cuantitativo. 


\section{Bibliografía}

Astin, J. A. (1998). Why patients use alternative medicine: results of a national study. Jama, 279(19), $1548-1553$.

Aurélie, A. (2007). La Vía Campesina. La globalización y el poder del campesinado. Madrid: Editorial Popular Madrid.

Bachrach, L. L. (1996). Deinstitutionalisation: promises, problems and prospects. In H. C. Knusden, \& G. Thornicroft (Eds.), Mental health service evaluation (pp. 3-18). Cambridge: Cambridge Universtiy Press.

Badia, A., \& Valldeperas, N. (2015). El valor histórico y estético del paisaje: claves para entender la vulnerabilidad de la interfaz urbano-forestal frente a los incendios. Scripta Nova, XIX(521). Retrieved from http://revistes.ub.edu/index.php/ScriptaNova/article/view/15125/18328

Ballvé, J. L. (2003). ¿Quién utiliza las medicinas no convencionales y por qué? Humanitas, 1, 3140.

Bauman, Z. (2013). Liquid times: Living in an age of uncertainty. Cambridge: John Wiley \& Sons.

Bowler, I. R. (1996). Agricultural land use and the post-productivist transition. In A. López, \& F. Molinero (Eds.), La investigación hispano-británica reciente en Geografía Rural: del campo tradicional a la transición postproductivista (pp. 7-31). Murcia: Asociación de Geógrafos Españoles.

Bragg, R. (2014). Nature-based interventions for mental wellbeing and sustainable behaviour: the potential for green care in the UK (Doctoral dissertation, University of Essex).

Carbone, A., Gaito, M., \& Senni, S. (2007, February 15-17). Consumers' Buying Groups in the Short Food Chains: Alternatives for Trust. Contribution presented at the 1st International European Forum on Innovation and System Dynamics in Food Networks, Innsbruck-Igls, Austria.

Cuéllar, M., Calle, A., \& Gallar, D. (Eds.) (2013). Procesos hacia la soberanía alimentaria. Perspectivas y prácticas desde la agroecología política. Barcelona: Icaria.

Da Silva, N. (2015). Estudo dos benefícios sociais, ambientais e económicos das hortas sociais biológicas do município da Póvoa de Lanhoso (Master dissertation, Instituto Politecnico do Viana do Castelo, Viana do Castelo).

Dessein, J., \& Bock, B. B. (Eds.) (2010). The Economics of Green Care in Agriculture (Report from COST Action 866, Green Care in Agriculture). Loughborough: Loughborough University. 
Dessein, J., Bock, B. B., \& De Krom, M.P. (2013). Investigating the limits of multifunctional agriculture as the dominant frame for Green Care in agriculture in Flanders and the Netherlands. Journal of Rural Studies, 32, 50-59.

Di lacovo, F. (2010). L'agricoltura sociale: pratiche e paradigmi nello scenario comunitario. Contribution presented at XLVII Convegno di Studi de la Società Italiana di Economia Agraria, Università degli Studi del Molise, Campobasso.

Di lacovo, F., \& O'Connor, D. (Eds.) (2009). Supporting Policies for Social Farming in Europe. Progressing Multifunctionality in Responsive Rural Areas. Firenze: ARISA.

Di lacovo, F., Moruzzo, R., Rossignoli, C., \& Scarpellini, P. (2014, March 24). Innovating rural welfare in the context of civicness, subsidiarity and co-production: Social Farming. Contribution presented at the 3rd EURUFU Scientific Conference, Social Issues and Health Care in Rural Areas, Sondershausen, Germany.

Di lacovo, F., Moruzzo, R., Rossignoli, C., \& scarpellini, P. (2014). Transition management and social Innovation in rural areas: Lessons from social farming. The Journal of Agricultural Education and Extension, 20, 327-347.

Duch, G. (2010). Lo que hay que tragar: minienciclopedia de política y alimentación. Barcelona: Los libros del lince.

Dupuis, E. M., \& Goodman, D. (2005). Should we go "home" to eat? Toward a reflexive politics of localism. Journal of Rural Studies, 21, 359-371.

Estela, O. (2015). Politiche pubbliche per economie locali resilienti, Territorio della Ricerca su Insediamenti e Ambiente. Rivista internazionale di cultura urbanistica, 15, 81-94.

Esping-Andersen, G. (Ed.) (1996). Welfare states in transition: national adaptations in global economies. London: Sage Publications.

Faus, P.; Blanco, E., \& Poitras, J. (2012): La ciudad jubilada: breve diccionario sobre los huertos informales en los ríos de Barcelona. Barcelona: P. Faus.

Fazzi, L. (2010). Social Co-operatives and Social Farming in Italy. Sociologia ruralis, 51(2), 119136.

Fernández, J. L., \& Morán, N. (2011). Huertos Comunitarios. El Ecologista, 70, 23-26.

Finoula R., \& Pascale A. (2008). L'agricoltura sociale nelle politiche pubbliche. Roma: INEA.

Firmino, A. (2011). Agricultura Social em Portugal-inovação de futuro incerto? Contribution presented at the I Encontro de Agricultura Social, Escola Superior Agrária de Coimbra. 
Galiakis, D. (2014). Typologies of Social Farming activities in Greece (PowePoint, Report for Europe for Citizens Programme, Project revaluating rural areas in Europe through the development of social farming activities, Ruract). Greece, Municipality of Agia. Retrieved from: $\quad$ http://www.dimosagias.gr/component/docman/doc_download/3752-ruract-campobasso.html

García-Llorente, M., Rossignoli, C., Moruzzo, R., \& Di lacovo, F. (2015, September 14-17). Social Farming practices to promote social-ecological sustainability in rural areas. Contribution presented at the Second International Conference on Agriculture in an Urbanizing Society, Rome, Italy.

Gerlach-Spriggs, N., Kaufman, R. E., \& Warner Jr, S. B. (2004). Restorative gardens: The healing landscape. New Haven: Yale University Press.

Goodman, D. (2004). Rural Europe redux? Reflections on alternative agroffood networks and paradigm change. Sociologia rurales, 44, 3-16.

Guidonet, A. (2010). ¿ Miedo a comer? Crisis alimentaria en contextos de abundancia. Barcelona: Editorial Icaria.

Guillén, A. M., González-Begega, S., \& Luque, D. (2015). Austeridad y ajustes sociales en el sur de Europa. La fragmentación del modelo de bienestar mediterráneo. In Federación Española de Sociología.

Retrieved from http://www.fes-sociologia.com/uploads/public/DEBATE\%20n25\%20Prensa\%20112.pdf

Guinjoan, E., Badia, A., \& Tulla, A.F. (2016). El nuevo paradigma de desarrollo rural. Reflexión teórica y reconceptualización a partir de la Rural Web. Boletín de la Asociación de Geógrafos Españoles, 71, 179-204.

Guirado, C., Badia, A., Tulla, A. F., Vera, A., \& Valldeperas, N. (2013). L'agricultura social. Aproximació conceptual i dinàmica en el context europeu. Biblio 3W, XVIII(1046), 1-27.

Guirado, C., Badia, A., Tulla, A. F., Vera, A., \& Valldeperas, N. (2014). La agricultura social en Catalunya: innovación social y dinamización agroecológica para la ocupación de personas en riesgo de exclusión. Ager, 17, 65-97.

Guirado,C., Valldeperas, N., Tulla, A. F., Sendra, L., Badia, A., Evard, C., Cebollada, A., Espluga, J., Pallarès, I., \& Vera, A. (2017). Social farming in Catalonia: Rural local development, employment opportunities and empowerment for people at risk of social exclusion. Journal of Rural Studies, 56, 180-197.

Hassink, J. (2003). Combining Agricultural Production and Care for Persons with Disabilities: a New Role of Agriculture and Farm Animals. Wageningen: Wageningen University. 
Hassink, J., \& Van Dijk, M. (Eds.) (2006). Farming for Health: Green-care Farming across Europe and the United States of America. Dordrecht: Springer.

Haubenhofer, D. K., Elings, M., Hassink, J., \& Hine, R. E. (2010). The development of green care in Western European countries. Explore, 6, 106-111.

Hine, R., Peacock, J., \& Pretty, J. (2008a). Care farming in the UK: contexts, benefits and links with therapeutic communities. International Journal Therapeuthic Communities, 29, 245-260.

Hine, R. A., Peacock, J. \& Pretty, J. (2008b). Green Spaces: Measuring the Benefits. Report for the National Trust. National Trust: Swindon.

Idescat (2013). Encuesta sobre la estructura de las explotaciones agrarias, INE, 1993-2013. INE: Barcelona

Idescat (2015a). Encuesta de población activa del INE. Cuarto trimestre de 2015. INE: Barcelona. Idescat (2015b). Encuesta de población activa del INE. Población ocupada por sectores de actividad en Cataluña, tercer trimestre de 2015. INE: Barcelona.

Idescat (2016). Padrón municipal de habitantes. INE: Barcelona.

Iranzo-García, E.; Pascual, J. A.; Blasco, C., \& Fansa, G. (2015, July 27-August 2). Accessible landscapes and health: A proposal for a comprehensive development of the rural landscape and heritage resources for therapeutic purposes. Contribution presented at the $23^{\text {rd }}$ Annual Colloquium Sustainable Rural Systems: Smart Answers for a Smiling Future Lisboa-Porto, Portugal.

Kuo, M. (2015). How might contact with nature promote human health? Promising mechanisms and a possible central pathway. Frontiers in Psycholgy, 6, 1093.

Lamarca, A. (2015). Agricultura Social: experiencias en la campiña sur de Córdoba. Córdoba: Universidad de Córdoba.

Lamarca, A.; Cúellar, M., \& Hernández, M. (2016, June 29-july 1). La agricultura social como herramienta de inclusión social y desarrollo local: un acercamiento a la Provincia de Córdoba, Andalucía. Contribution presented at the III Congreso Internacional de Estudios del Desarrollo, Universidad de Zaragoza, Zaragoza.

Lamb, R., \& Bachrach, L. (2001). Some Perspectives on Deinstitutionalization. Psychiatric Services, $52,1039-1045$.

Laparra, M., \& Pérez, B. (Coord.) (2012). Crisis y fractura social en Europa. Causas y efectos en España. Barcelona: Obra Social "La Caixa".

Laville, J. L. (2015). Asociarse para el bien común. Tercer Sector, Economía Social y Economía Solidaria. Barcelona: Icaria editorial. 
Leck, C., Evans, N., \& Upton, D. (2014). Agriculture - who cares? An investigation of 'Care Farming' in the UK. Journal of Rural Studies, 34, 313-325.

Levenstein, H. (2012). Fear of Food: A History of why We Worry about what We Eat. Chicago: University of Chicago Press.

Lockwood, J. A. (1999). Agriculture and biodiversity: finding our place in this world. Agriculture and Human Values, 16, 365-379.

Lohrberg, F. (2016). Urban Agriculture Europe: Agriculture Interacting with the Urban Sphere. In F. Lohrberg, L. Licka, L. Scazzosi, \& A. Timpe (Eds.), COST Action Urban Agriculture Europe (pp. 813). Berlin: Jovis VerlagGmbH.

Lopez-Moreno, I., Monllor, N., Guillamon, J., Guirado, C., \& Medina, F. X. (2016). La cuina compromesa: estudio de caso exploratorio sobre la viabilidad de prácticas alternativas de restauración en la nueva ruralidad catalana. Documents d'Anàlisi Geogràfica, 62(3), 595-612.

López, J. A., \& Fernández, D. (2003). Con la comida no se juega: Alternativas autogestionarias a la globalización capitalista desde la agroecología y el consumo. Madrid: Traficantes de Sueños.

Maarse, H. (2006). The privatization of health care in Europe: an eight-country analysis. Journal of Health Politics, Policy and Law, 31, 981-1014.

Marbán, V., \& Rodríguez, G. (2006). Estado de bienestar y tercer sector social en España. El estado de la investigación social. CIRIEC-España, 56, 117-139.

Matarán Ruiz, A. (2013) Participación social en la protección activa de los espacios agrarios periurbanos: un estado de la cuestión. Boletín de la Asociación de Geógrafos Españoles, 63, 5779.

Medina, F. X. (2010). Alimentació i pors alimentàries: la crisi de les vaques boges (EEB) des d'una perspectiva local. In F. X. Medina (Ed.), Reflexions sobre les alimentacions contemporànies. De les biotecnologies als productes ecològics (pp. 47-72). Barcelona: Editorial UOC.

Miele, M. (2006). Consumption culture: The case of food. In P. J. Cloke, T. Marsden, \& P. Mooney (Eds.), Handbook of Rural Studies (pp. 344-354). London: SAGE Publications.

Monllor, N. (2013). La nova pagesia: vers un nou model agrosocial. Quaderns Agraris, 35, 7-24. Mourão, I., \& Brito, L. M. (2013). Horticultura Social e Terapêutica. Hortas urbanas e atividades com plantas no modo de produção biológico. Porto: Publindústria.

Murdoch, J., \& Miele, M. (1999). Back to nature: Changing worlds of production in the food sector. Sociologia ruralis, 39, 465-483.

Naldini, M. (2003). The family in the Mediterranean welfare states. London: Routledge. 
O'connor, D. Lai, M., \& Watson, S. (2010). Overview of social farming and rural development policy in selected EU member states, NRN Joint Thematic Initiative on Social Farming. Report by the European Network for Rural Development.

Palinkas, L. A., Kabongo, M. L., \& San Diego Unified Practice Research In Family Medicine Network (2000). The use of complementary and alternative medicine by primary care patients. Journal of Family Practice, 49, 1121-1132.

Pavolini, E., León, M., Guillén, A. M., \& Ascoli, U. (2015). From austerity to permanent strain? The EU and welfare state reform in Italy and Spain. Comparative European Politics, 13(1), 56-76.

Petrini, C. (2003). Slow food: the case for taste. New York: Columbia University Press.

Ploeg, J. D. (2008). The new peasantries: struggles for autonomy and sustainability in an era of empire and globalization. London: Routledge.

Ploeg, J. D. (2010). The food crisis, industrialized farming and the imperial regime. Journal of Agrarian Change, 10, 98-106.

Pomar, A., \& Tendero, G. (2015). Ja volem el pa sencer. Respostes a la pobresa alimentària en clau de Sobirania Alimentària. Barcelona: Aliança per la Soberania Alimentària de Catalunya (ASAC).

Relf, P. D. (2006). Agriculture and Health care. The care of plants and animals for therapy and rehabilitation in the United States. In J. Hassink, \& M. van Dijk (Eds.), Farming for Health: Greencare farming across Europe and the USA, vol. 3 (pp. 309-343). Dordrecht: Springer.

Renting, H., Marsden, T., \& Banks, J. (2003). Understanding alternative food networks: exploring the role of short food supply chains in rural development. Environment and planning, A(35), 393412.

Riera, C. (2009). Serveis socials, acció comunitària i participació ciutadana: un nou marc per a la inclusió. Nous escenaris, reptes, estratègia i metodologia (Papers d'Acció Social, 10). Retrieved from http://xarxanet.org/sites/default/files/gencat-serveissocials.pdf

Saltman, R., Busse, R., \& Figueras, J. (2006). Decentralization in health care: strategies and outcomes. London: McGraw-Hill Education.

Sánchez Hernández, \& J. L. (2009). Redes alimentarias alternativas: concepto, tipología y adecuación a la realidad española. Boletín de la Asociación de Geógrafos Españoles, 49, 185207.

Schneider, S. (2008). Good, Clean, Fair: The Rhetoric of the Slow Food Movement. College English, 70(4), 384-402. 
Sempik, J., Hine, R., \& Wilcox, D. (Eds.) (2010). Green Care: A Conceptual Framework (Report of the Working Group on the Health Benefits of Green Care, COST 866, Green Care in Agriculture). Loughborough: Loughborough University.

Sevilla, E. (2006). De la sociología rural a la agroecología. Barcelona: Icaria Editorial.

Stigsdotter, U. K., Palsdottir, A. M., Burls, A., Chermaz, A., Ferrini, F., \& Grahn, P. (2011). Naturebased therapeutic interventions. In K. Nilsson, M. Sangster, C. Gallis, T. Hartig, S. Vries, K. Seeland, \& J. Schipperijn (Eds.), Forests, trees and human health (pp. 309-342). Netherlands: Springer.

Townsend, M. (2006). Feel blue? Touch green! Participation in forest/woodland management as a treatment for depression. Urban Forestry \& Urban Greening, 5, 111-120.

Tulla, A. F. (Coord.) (2014). L'Agricultura Social en el desenvolupament local i l'ocupació de col•lectius en risc de marginació (Results report of the research projecct ACUP2011-00023, Programa RecerCaixa, convocatoria 2011). Retrieved from

https://agriculturasocialcat.wordpress.com/resultats/

Tulla, A. F., Vera, A., Badia, A., Guirado, C., \& Valldeperas, N. (2014). Rural and Regional Development Policies in Europe: Social Farming in the Common Strategic Framework (Horizon 2020). Journal of Urban and Regional Analysis, VI(1), 35-52.

Tulla, A. F., Guirado, C., Badia, A., Vera, A., Valldeperas, N., \& Evard, C. (2015). La agricultura social a Catalunya. Una doble alternativa: Desenvolupament local i ocupació de col•lectius en risc d'exclusió social. Quaderns Agraris, 38, 23-49.

Tulla, A. F., Vera, A., Valldeperas, N., \& Guirado, C. (2017) New approaches to sustainable rural development: Social Farming as an opportunity in Europe? Human Geographies, 11(1), 25-40.

Vivas, E. (2010). El consum agroecològic a l'Estat espanyol: una opció política. In F. X. Medina (Ed.), Reflexions sobre les alimentacions contemporànies (pp. 159-174). De les biotecnologies als productes ecològics. Barcelona: Editorial UOC.

Walker, B., \& Salt, D. (2006). Resilience thinking: Sustaining ecosystems and people in a changing world. Washington D. C.: Island Press.

Willems, J. (2013). Opinion of the European Economic and Social Committee on "Social farming": green care and social and health policies (Official Journal of the European Union 2013/C44/07). Retrieved from

http://eur-lex.europa.eu/legal-

content/EN/TXT/?uri=uriserv:OJ.C_.2013.044.01.0044.01.ENG\&toc=0]:C:2013:044:TOC 